\title{
Subcortical Atlas of the Rhesus Macaque (SARM) for Magnetic Resonance Imaging
}

\author{
Renée Hartig ${ }^{\mathrm{a}, \mathrm{b}, \mathrm{c}}$, Daniel Glen ${ }^{\mathrm{d}}$, Benjamin Jung ${ }^{\mathrm{e}, \mathrm{f}}$, Nikos K. Logothetis ${ }^{\mathrm{b}, \mathrm{g}}$, George Paxinos ${ }^{\mathrm{h}}$, \\ Eduardo A. Garza-Villarreal', Adam Messinger ${ }^{\dagger}$, Henry C. Evrard ${ }^{a, b, j}$
}

a Werner Reichardt Centre for Integrative Neuroscience, University of Tübingen, Tübingen, Germany

${ }^{\mathrm{b}}$ Max Planck Institute for Biological Cybernetics, Tübingen, Germany

${ }^{\mathrm{c}}$ Department of Psychiatry and Psychotherapy, Central Institute of Mental Health, Medical Faculty Mannheim, Heidelberg University, Mannheim, Germany

${ }^{\mathrm{d}}$ Scientific and Statistical Computing Core, National Institute of Mental Health, Bethesda, USA

e Department of Neuroscience, Brown University, Providence RI USA

${ }^{f}$ Laboratory of Brain and Cognition, National Institute of Mental Health, Bethesda, USA

${ }^{g}$ University of Manchester, Manchester, United Kingdom

${ }^{\mathrm{h}}$ Neuroscience Research Australia and The University of New South Wales, Sydney, NSW 2031, Australia

'Instituto de Neurobiologia, Universidad Nacional Autónoma de México campus Juriquilla, Queretaro, Mexico

j Center for Biomedical Imaging and Neuromodulation (C-BIN), Nathan S. Kline Institute for Psychiatric Research, Orangeburg, New York, USA

Corresponding author:

Henry C. Evrard

Henry.Evrard@nki.rfmh.org, Henry.Evrard@tuebingen.mpg.de, Henry.Evrard@cin.uni-

tuebingen.de

Nathan S. Kline Institute for Psychiatric Research

140 Old Orangeburg Road

Orangeburg, New York 10962, USA

\section{Highlights}

- We present the Subcortical Atlas of the Rhesus Macaque (SARM).

- SARM provides a neuroanatomical reference frame for neuroimaging analysis.

- The entire subcortex is mapped, including the thalamus, basal ganglia, and brainstem.

- ROls are grouped hierarchically, making SARM useful at multiple spatial resolutions.

- SARM is in the NMT v2 template space and complements the CHARM atlas for the cortex. 


\section{Abstract}

40 Digitized neuroanatomical atlases are crucial for localizing brain structures and analyzing 41 functional networks identified by magnetic resonance imaging (MRI). To aid in MRI data 42 analysis, we have created a comprehensive parcellation of the rhesus macaque subcortex using 43 a high-resolution ex vivo structural imaging scan. The structural scan and its parcellation were 44 warped to the updated NIMH Macaque Template (NMT v2), an in vivo population template, 45 where the parcellation was refined to produce the Subcortical Atlas of the Rhesus Macaque 46 (SARM). The subcortical parcellation and nomenclature reflect those of the $4^{\text {th }}$ edition of the 47 Rhesus Monkey Brain in Stereotaxic Coordinates (RMBSC4; Paxinos et al., in preparation). The 48 SARM features six parcellation levels, arranged hierarchically from fine regions-of-interest 49 (ROIs) to broader composite regions, suited for fMRI studies. As a test, we ran a functional 50 localizer for the dorsal lateral geniculate (DLG) nucleus in three macaques and found significant $51 \mathrm{fMRI}$ activation in this atlas region. The SARM has been made openly available to the 52 neuroimaging community and can easily be used with common MR data processing software, 53 such as AFNI, where the atlas can be embedded into the software alongside cortical macaque 54 atlases.

\section{Keywords}

segmentation; fMRI; anatomy; cerebellum; thalamus; brainstem

\section{Abbreviations}

58 fMRI functional Magnetic Resonance Imaging

59 DLG Dorsal Lateral Geniculate

60 NHP non-human primate

$61 \quad$ NMT v2 NIMH Macaque Template

62 RMBSC4 $4^{\text {th }}$ edition of the Rhesus Monkey Brain in Stereotaxic Coordinates

63 ROls regions of interest

64 SARM Subcortical Atlas of the Rhesus Macaque

65 CHARM Cortical Hierarchy Atlas of the Rhesus Macaque 


\section{Introduction}

67 As functional magnetic resonance imaging (fMRI) continues to advance spatiotemporal

68 resolution limits, there is a growing opportunity for researchers to examine subcortical regions

69 and their involvement in cortico-subcortical networks. These smaller subcortical regions have,

70 however, largely been absent from digitized atlases applicable to MRI research with non-human

71 primates (NHPs). In contrast to human research, where several subcortical atlases exist, NHP

72 researchers typically have to employ workarounds and parcellate individual regions of interest

73 (ROIs) themselves. To address this void, we present the Subcortical Atlas of the Rhesus

74 Macaque (SARM), a digital subcortical atlas offering a standardized parcellation for ROI and

75 network analyses.

The development of the SARM is timely. While previously used in only a few primate research centers, $\mathrm{fMRI}$ is now being employed in many NHP laboratories (Milham et al., 2018). The use of contrast agents, improved sequences, and high-field magnets is increasing the signal-to-noise ratio and spatial resolution into the realm where subcortical activations can be reproducibly detected (e.g., Baker et al., 2006; Ortiz-Rios et al., 2015; Quan et al., 2020). Technological improvements in data collection methods have also resulted in greater potential

82 for employing fMRI concurrently with subcortical electrical microstimulation (Logothetis et al., 83 2010; Arsenault \& Vanduffel, 2019; Murris, Arsenault \& Vanduffel, 2020), optogenetics (Nassi et 84 al., 2015; Klein et al., 2016; Stauffer et al., 2016), or electrophysiological recordings (Logothetis 85 et al., 2012), all the while capturing the mesoscopic and systems-level effects (see also, Klink et 86 al., this issue). Such studies require fine-grain delineations of the subcortex to aid both in 87 planning stereotaxic implantations and interpreting local signal modulation. Finally, while NHP $88 \mathrm{fMRI}$ still typically relies on two or three subjects, there is a growing interest in using larger 89 groups and applying group analyses (e.g. Fox et al., 2018). The advent of multi-center data 90 sharing (Milham et al., 2018) also allows for the possibility of larger sample sizes, and clearly 91 calls for group-level analyses performed on data aligned to a population brain template with 92 standardized atlases (Milham et al., 2020; Jung et al., this issue).

Previous NHP studies examining subcortical activity have created their own individual 94 masks covering regions known to include specific brainstem nuclei. For example, Logothetis et 95 al. (2012) manually segmented 25 subcortical ROls for each of their five subjects separately. 96 Noonan et al. (2014) masked the area between the medulla and midbrain for localizing activity 97 from serotonergic nuclei with $0.5 \mathrm{~mm}$ spatial resolution. Murris et al. (2020) registered their 
98 functional data to the D99 macaque template (Reveley et al., 2017) and then added ROls for

99 the ventral tegmental area (VTA) and accumbens nucleus (Acb), which are absent in the D99

100 atlas. While creating individual masks is one strategy to study regional fMRI activity, precise

101 delineation of structural boundaries requires not only high-quality structural scans but a great

102 deal of labor and anatomical expertise. Furthermore, for comparisons across individuals or

103 group-level analysis, single-subject scans and regional masks must then be nonlinearly

104 registered to a common reference template. While warping of $\mathrm{fMRI}$ data to a standard space

105 can be successful for fairly large subcortical parcellations (e.g., the hippocampus, amygdala,

106 and distinguishable midbrain regions; Fox et al., 2015), nonlinear registration of smaller

107 subcortical structures can be a delicate step in the data processing pipeline. The SARM allows

108 for varying alignment and resolution limits, providing hierarchically arranged groupings of

109 regions that are suited for different purposes, including functional neuroimaging studies.

In comparison to human MRI brain atlases (e.g., Accolla et al., 2014; Pipitone et al., 2014; Ewert et al., 2017; Pauli et al., 2018), and despite the existence of NHP paper atlases

112 including an exhaustive mapping of the subcortex (Paxinos et al., 2009; Martin \& Bowden,

113 2000), limited efforts have been made to digitize the macaque subcortex parcellations. Previous

114 attempts to digitize subcortical parcellations from printed macaque atlases have provided some

115 segmentation of the subcortex. For example, the Saleem and Logothetis (2012) atlas was

116 digitized by alignment to a high-resolution MRI of an ex vivo surrogate (Reveley et al. 2017).

117 However, this digital D99 atlas includes only some subcortical structures (e.g., hippocampus,

118 amygdala, striatum, and claustrum). Likewise, the parcellation of post-mortem macaque brains

119 by Calabrese et al. (2015) contains a detailed segmentation of most telencephalic and

120 diencephalic brain nuclei (Paxinos et al., 2009) but little to no parcellation of the brainstem. The

121 NeuroMaps macaque atlas covers the whole brain and is presented on an ex vivo juvenile

122 rhesus macaque brain (Bakker, Tiesinga \& Kötter, 2015; Rohlfing et al., 2012). The NeuroMaps

123 segmentation was later refined on the INIA19 adult population in vivo symmetric template

124 (Rohlfing et al., 2012). Rohlfing and colleagues noted, however, that their segmentation of the

125 basal forebrain, hypothalamus and amygdala is incomplete and that the internal segmentation

126 of the thalamus, midbrain and hindbrain may not be reliable. Although a more detailed digital

127 subcortical map is needed, segmenting all the minute cytoarchitectonic subnuclei that can be

128 appreciated under the microscope would be of little value at fMRI resolution. Using an updated

129 version of the Rhesus Monkey Brain in Stereotaxic Coordinates (Paxinos et al., 2009; Paxinos

130 et al., in preparation) for guidance, the SARM addresses the need for a more comprehensive 
131 subcortical segmentation while attempting to strike a practical balance between anatomical

132 details and the constraints imposed by the lower anatomical resolution of MRI.

While ex vivo scans, such as the D99 surrogate or the Calabrese ex vivo population

134 template (Calabrese et al., 2015), can provide great detail because they are not impacted by 135 animal movement or physiological noise, in vivo templates better reflect the living brain's 136 configuration (e.g., with regard to size, ventricle shape, and the presence of cerebrospinal fluid). 137 Atlases drawn on a single subject template can precisely reflect the particular anatomy of that 138 subject but may not be morphologically representative of the species due to large inter139 individual variability. The SARM was fit to version 2 of the NIMH Macaque Template (NMT v2), 140 a high-resolution population template based on in vivo scans collected at high field strength (4.7 $141 \mathrm{~T})$ from a large cohort $(\mathrm{N}=31)$ of adult rhesus monkeys (Seidlitz et al, 2018; Jung et al., this 142 issue). The NMT v2 compares favorably to the INIA19 template in terms of resolution $(0.25 \mathrm{~mm}$ 143 vs. $0.50 \mathrm{~mm}$ isotropic), allowing for finer parcellation of subcortical structures, and is already 144 home to the Cortical Hierarchy Atlas of the Rhesus Macaque (CHARM; Jung et al., this issue). 145 Because average population templates are representative, most individuals will require 146 relatively little distortion to be aligned to such a template as compared to an ex vivo or individual 147 scan (Kochunov et al., 2001; Molfese et al., 2015; Feng et al., 2017). This, in effect, minimizes 148 alignment errors, which are of particular importance for small subcortical nuclei.

To create the SARM, we relied on the high resolution and precision of an ex vivo singlesubject structural scan and previously obtained histological material to draw the primary 152 they were manually refined to reflect the representative anatomy of the population template. The 153 SARM parcellation was then hierarchically grouped into larger composite structures to create 154 region of interest $(\mathrm{ROI})$ clusters suitable for (f)MRI analysis. The SARM is available on various

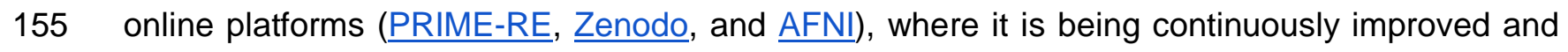
156 further delineated. 


\section{Materials \& Methods}

\subsection{Atlas Preparation}

\subsubsection{Ex Vivo Anatomical Sample}

A whole-brain ex vivo sample from one adult female rhesus macaque (G12; Macaca mulatta; 8 $\mathrm{kg}$ ) was used as a single-subject anatomical template to parcellate the subcortex. This subject was part of an anatomical study approved by the local authorities and in full compliance with the European Parliament and Council Directive 2010/63/EU. The subject was not involved in any invasive procedures and never underwent intracerebral surgery. After transcardial fixation with 4\% formalin (Evrard et al., 2012), the brain was placed into a jar of agar and positioned upright in a horizontal 7T Bruker BioSpec scanner, with the brain oriented parallel to the scanner (dorsal side positioned upward) (Bruker BioSpin, Ettlingen, Germany). The entire brain was scanned using a high-resolution fast low-angle shot (FLASH) sequence (voxel dimensions: 0.15x0.15x1.0 mm; flip angle: 50; TR/TE: 2500/9 msec; field-of-view (FOV): 70x52 mm; matrix size: 468x346; 78 coronal slices).

\subsubsection{Segmentation in Individual (G12) Space}

172 Subcortical ROls were manually drawn by author HCE onto coronal slices of the G12 high173 resolution ex vivo anatomical scan using the Amira software (Amira 6.0.1; FEI). The fine spatial

174 resolution of the contrast variation in the slices enabled recognizing and mapping discrete 175 anatomical regions identified in corresponding histological sections from the $4^{\text {th }}$ edition of the 176 Rhesus Monkey Brain in Stereotaxic Coordinates (RMBSC4; Paxinos et al., in preparation). The 177 order of the figures in this upcoming edition does not differ from the $2^{\text {nd }}$ edition; thus readers can 178 still refer to the printed second edition of RMBSC (Paxinos et al., 2009) when references to 179 specific figures are made in the text below. These reference sections previously underwent 180 Nissl and AChE staining (Paxinos et al., 2009), and were recently scanned using a slide181 scanner microscope (AxioScan; Zeiss) for further examination (Paxinos et al., in preparation). 182 The ROls were drawn while examining all three stereotaxic planes to reduce inconsistencies in 183 delineation across slices. Regions defined in RMBSC4 that were too small and not clearly 184 discernible from changes in contrast in the G12 scan were grouped together in larger ROls, as 185 detailed in the Results (Section 3.1). Additional resources included prior architectonic 186 parcellations of the hypothalamus (Saper et al., 2012), thalamus (Olszewki, 1952; Calzavara et 
al., 2005; Evrard and Craig, 2008; Mai and Forutan, 2012), amygdala (Amaral et al., 1992; Stefanacci et al., 2000), and basal ganglia (Haber et al., 2012).

\subsubsection{Nonlinear Registration}

The single-subject (G12) ex vivo structural scan and subcortical segmentation were nonlinearly registered to the symmetric NMT v2 full-head anatomical template for rhesus macaques. The NMT v2 template (Jung et al., this issue) is in stereotaxic orientation (Horsley and Clarke, 1908; also referred to as the Frankfurt Zero plan). The subcortical segmentation was refined on a single hemisphere (the left) of the NMT v2 and mirrored onto the opposite hemisphere in order to assure that the resulting parcellation has left and right ROls of equal size.

To coregister the G12 template and atlas to the NMT v2, the NIFTI images were first converted to MINC format (http://www.bic.mni.mcgill.ca/ServicesSoftware/MINC) and the origin of the spatial coordinates was adjusted to correspond to the intersection of the midsagittal section and the interaural line (i.e., ear bar zero, EBZ). Then, we used volmash and volflip (MINC widgets) to reorient the images to the NMT v2. The G12 template was then converted back to NIFTI. Using Advanced Normalization Tools (ANTs; version 2.3.1.dev159-gea5a7; Avants et al., 2014), we made a negative image of the $\mathrm{G} 12$ so its contrast would be similar to the T1-weighted NMT v2 template. The G12 template showed air bubble-induced artifacts around the left lateral ventricle that affected registration. To correct these artifacts and improve registration, we manually traced each artifact to the underlying tissue (namely, the putamen) and matched it with the tissue's intensity. This new volume was then corrected for N4 Bias Field artifacts (Tustison et al., 2010). The ANTs registration pipeline was optimized using an in-house script that employed a custom mask of the subcortex for some of the registration steps. After computing the G12 to NMT v2 template registration, we used antsApplyTransformation to nonlinearly coregister the subcortical parcellation to the NMT v2 with Generic Label 211 interpolation.

\subsubsection{Refinement of ROIs in the NMT v2 Template}

213 The resulting atlas regions suffered from some irregularities stemming from the limitations of the 214 original anisotropic voxels (high resolution within the coronal plane, but coarser resolution 215 across planes) and from the interpolation methods associated with the nonlinear warp of the 216 ROI labels. Therefore, we followed the ANTs-based alignment pipeline with a procedure to 217 spatially regularize regions using AFNI commands. The regions were processed with a modal 
smoothing technique that replaces each voxel with the most common label in a 1- or 2-voxel spherical neighborhood around every voxel. A select list of thin or small regions were smoothed using the 1 voxel mode, and all other regions were smoothed using the 2 voxel mode. The data were masked by the CSF and blood vessel segmentations from NMT v2. Each ROI was automatically further refined by examining the distribution of voxel intensities in NMT v2. For each ROI, we sampled voxel intensities of NMT v2 in that ROI, and voxels farther than three standard deviations away from the mean intensity (potentially indicating encroachment of the $\mathrm{ROI}$ into a different tissue class) were compared with eight neighboring voxels and reassigned to the label of the voxel with the most similar intensity. This outlier detection was performed across ten iterations. The quality of the alignment between the transformed G12 and the NMT v2 template was assessed by viewing the former on the outline of the latter using @chauffeur_afni. Finally, the atlas was assessed for discontinuities, and discontinuous clusters smaller than five percent of the size of the largest portion of the ROI were replaced with labels from neighboring voxels. With the atlas regions now transformed to the NMT v2 symmetric template space, the regions were manually adjusted, again in Amira by author HCE, with reviewing by authors HCE and GP, to reflect the anatomical transitions evident in this population template. Before exporting from AMIRA, a Gaussian smoothing (2x2x2 pixel filter mask) was applied across the 3D volume using the "Smooth Labels" function. Following AMIRA export, the SARM regions were modally smoothed with a 1.8 voxel radius and discontinuous clusters smaller than five percent of the size of the largest portion of the ROI were again replaced with labels from neighboring voxels. At each step, volume changes of each ROI were tracked to prevent large, unintended changes to the ROls.

\subsection{Subcortical Naming Hierarchy}

\subsubsection{Hierarchical Grouping}

242 To create ROls of varying spatial resolution, the neighboring regions in the primary parcellation 243 were iteratively grouped to form a hierarchy of subcortical structures across six levels that 244 describes progressively larger and more general anatomical regions. This hierarchy forms the 245 SARM. The finest level of the SARM hierarchy (level 6) individually itemizes each of our 246 manually drawn ROls, which were defined on the basis of the RMBSC4, as described above.

247 Composite regions in levels 1-5 were successively built from smaller adjacent areas in the next 248 finer level. While the brain can most broadly be subdivided into the forebrain, midbrain, and 249 hindbrain, level 1 begins with the developmental and embryological sub-divisions of the 
subcortex, namely the tel-, di-, mes-, met-, and myel-encephalon. The SARM levels 2-4 consist of ROls of sufficient size to accommodate functional imaging voxels that are typically 1.25-1.50 $\mathrm{mm}$ on a side, whereas levels 5-6 ROls may benefit from the higher resolution of structural imaging. Levels 5 and 6 of the SARM were left largely similar to allow for potential future delineation of SARM regions. In most cases, we grouped the ROls based on their developmental and/or functional relationships (Mai and Paxinos, 2012; Puelles et al., 2013; see also Calabrese et al., 2015 for a similar approach), with the condition that these ROls had to be spatially contiguous. In other cases, in particular at coarser levels, ROls had to be grouped solely based on their spatial proximity.

Independent of their hierarchical classification, all ROls were classified as being primarily subcortical gray matter or white matter. In select instances, ROls composed primarily of white matter or other tissue types were included in larger composite ROls to make them whole (e.g. the internal capsule was included in the striatum to bridge the caudate and putamen) and because sparse cell bodies within such white matter regions can lead to their

264 functional activation.

\subsubsection{Nomenclature}

266 Each ROI and group of ROls has a unique full name and abbreviation. At levels 5 and 6 , the 267 names and abbreviations of the ROls typically match those defined in the RMBSC4, with some 268 exceptions (see Results) to accommodate the most commonly used naming convention in NHP $269 \mathrm{fMRI}$. At levels 2 to 4, the names and abbreviations of the groups of ROls reflect either a common developmental origin (e.g., pallial vs. subpallial amygdala; Puelles et al., 2013), a classical neuroanatomical grouping (e.g., basal ganglia; Mai and Paxinos, 2012) or a spatial proximity (e.g., dorsal vs. ventral mesencephalon).

AFNI allows for flexible indexing of ROls by either index number, abbreviation, or the full name of the ROI. To prevent conflicts between index numbers and names, SARM abbreviations do not start with a number (e.g., the abducens nucleus is abbreviated 6N in RMBSC4 but N6 in SARM). In addition, to maximize compatibility with scripts and programs, abbreviations do not

277 include special characters, and full names use underscores in place of spaces. A full list of the 278 current SARM regions is provided in Supplementary Table 1. A spreadsheet of the hierarchy 279 and full list of SARM structures is also available for download with the NMT v2 package. 
281 To illustrate the usefulness of this atlas within the context of fMRI data analysis, a functional 282 localizer for the dorsal lateral geniculate nucleus (DLG) (also referred to as the lateral geniculate 283 nucleus, LGN) was included, from a larger experimental program, with three adult rhesus 284 macaques (Macaca mulatta; 1 female; average weight: $10.11 \mathrm{~kg}$ ). Experiments were conducted 285 following a previously described opiate-based anesthesia protocol (Logothetis et al., 2010). 286 Animals were treated according to the guidelines of the European Parliament and Council 287 Directive 2010/63/EU on the protection of animals used for experimental and other scientific 288 purposes. Experimental protocols were approved by the local German authorities.

\subsubsection{Image Acquisition}

290 Neuroimaging data were acquired using a vertical 7 Tesla NMR scanner (Bruker, Billerica, MA, 291 U.S.A.) and Paravision software (version 5). $\mathrm{fMRI}$ data were acquired with a quadrature coil and 292 double-shot gradient-echo echo planar imaging (GE-EPI; voxel dimensions: 0.75x0.75x2.0 mm; 293 flip angle: 53; TR/TE: 2000/19 msec; FOV: 96x96 mm; matrix size: 128x128; 20 axial slices). 294 Slice volumes were acquired contiguously. During each experiment, a T2-weighted rapid 295 acquisition with relaxation enhancement (RARE) scan was collected to image the native 296 structural space (RARE factor: 8; voxel dimensions: $0.375 \times 0.375 \times 1.0 \mathrm{~mm}$; flip angle: $180^{\circ}$; 297 TR/TE: 6500-8500/16 msec; FOV: 96x96 mm; matrix size: 256x256; 40 axial slices). Acquired 298 data were converted offline from Bruker file format to 4D NIFTI files using the Unix-based 299 pvconv.

\subsubsection{Stimulus}

301 A flickering checkerboard stimulus was visually presented (Logothetis et al., 1999) during a 10 302 minute GE-EPI scan, consisting of 300 volumes. The stimulus was presented for $4 \mathrm{sec}$ 303 preceded by an 8 sec OFF period, and followed by a longer 18 sec OFF period, allowing return 304 of the blood-oxygen-level-dependent (BOLD) signal to baseline. Two sessions per subject were 305 collected and analyzed using two common software packages (SPM \& AFNI) to validate the 306 application of SARM for studying subcortical activity across different processing pipelines.

\section{2.3.3 SPM-Based Image Analysis}

308 Functional data were realigned using SPM12 (Statistical Parametric Mapping; Wellcome 309 Department of Imaging Neuroscience, London, UK) to obtain six rigid-body transformation 
310 parameters and then aligned to each subject's native anatomical (RARE) scan. Each subject's

311 RARE was subsequently translated to the NMT v2 space, and this linear transformation was

312 applied to all relevant functional scans. Data were nonlinearly aligned using SPM-based Dartels,

313 a diffeomorphic warping algorithm (Ashburner, 2007), which relies on tissue class identification

314 and segmentation. The resulting deformation matrix was applied to each individual's RARE and

$315 \mathrm{fMRI}$ images. At each step, the spatial alignment was checked by direct visual examination. The

316 EPIs were smoothed (2 mm FWHM Gaussian) and the fMRI data were estimated using a

317 General Linear Model (GLM), which included as regressors the rigid-body transformation

318 parameters, in the event-related responses correlated with the visual stimulus presentation $\left(B_{1}\right)$

319 and the baseline activity $\left(B_{0}\right)$. The fMRI data were averaged across sessions for each subject,

320 and significant activations were assessed with a T-contrast ( $p<0.05$, FDR-corrected).

\section{$321 \quad$ 2.3.4 AFNI-Based Image Analysis}

322 Using AFNI (Cox, 1996), functional data were processed by first computing the alignment of 323 each subject's T2 structural (RARE) scan to the NMT v2 template using the @animal_warper 324 pipeline (Jung et al., this issue). To address the contrast (e.g., of CSF, GM and WM) profile 325 inversion between NMT v2 and the functional localizer datasets, we used an alignment method 326 that identifies local negative correlations to minimize the cost function, the Local Pearson 327 Correlation (Ipc; Saad, 2009). This cost function was used for both affine and nonlinear 328 alignment. The alignment was assessed using AFNI visualization tools. The affine and nonlinear 329 transformations and the skull-stripped dataset served as the input to the functional processing 330 performed by afni_proc.py. This processing used typical options for motion correction, alignment 331 of the functional data to the individual's T2 anatomical dataset, and modal smoothing (by 1 332 voxel), followed by a per-voxel mean scaling. The normalized functional data were interpolated 333 to an isotropic voxel resolution of $1.25 \mathrm{~mm}^{3}$. The functional paradigm was modeled using a 334 BLOCK hemodynamic response function model, stimuli convolved with a 4-sec duration boxcar 335 function and normalized to unit size.

\subsection{Data Accessibility and Availability}

337 The SARM and NMT v2 files are provided in NifTI and GifTI file format for compatibility with 338 most neuroimaging programs. This package also includes: the original G12 dataset with ROI 339 drawings in their original space and the full list of SARM ROls, abbreviations, and grouping 340 levels. For data transformation and analysis, relevant scripts are also provided. All resources 
341 described are currently openly available or will be made available in the near future through the

342 PRIME-Resource Exchange (https://prime-re.github.io/) (Messinger et al., this issue), Zenodo

343 (https://zenodo.org/record/4026520\#.X10X95P0nlw), and can be downloaded along with the 344 NMT $\quad$ v2 from the AFNI website

345 (https://afni.nimh.nih.gov/pub/dist/doc/htmldoc/nonhuman/macaque tempatl/atlas sarm.html) or 346 using the AFNI command @Install_NMT.

\section{Results}

\subsection{Subcortical ROI Segmentation and Hierarchical Grouping}

349 This first version of the SARM (SARM v1) contains 206 primary subcortical ROls. These ROls were first drawn on the G12 high-resolution scan and then nonlinearly aligned to the NMT v2 population-averaged symmetrical template. Most of these ROls were anatomically identifiable in G12 and, to some extent, in NMT v2, based on local signal contrast variations. Regions likely to on their most likely topological localization and neighborhood relationships, using RMBSC4 as the principal reference (Paxinos et al., 2009; Paxinos et al., in preparation). Individual ROls represent either a single homogeneous anatomical entity, as defined in RMBSC4, or a collection of smaller cytoarchitectonic entities that could not be distinguished from one another due to a lack of contrast differentiation. Beyond the definition of the manually drawn primary ROls, we created ROls of progressively larger size by successively aggregating primary ROls across six hierarchical levels. These six levels can accommodate structural and functional neuroimaging datasets of various spatial resolutions and analyses at different degrees of anatomical detail. The following sections report, successively, the alignment of G12 to NMT v2 (Section 3.1.1), general observations on the hierarchical grouping of the ROls (Section 3.1.2), and, finally, an overview of the definition of the individual ROls and their hierarchical groups (Section 3.1.3).

\subsubsection{Alignment of G12 to NMT v2}

Figure 1 portrays the G12 ex vivo anatomical MRI in stereotaxic space, corresponding to the NMT v2 template at 5 representative coronal sections, where the nonlinear alignment computed using ANTs was overlaid onto the edge contours of the NMT v2 template. 


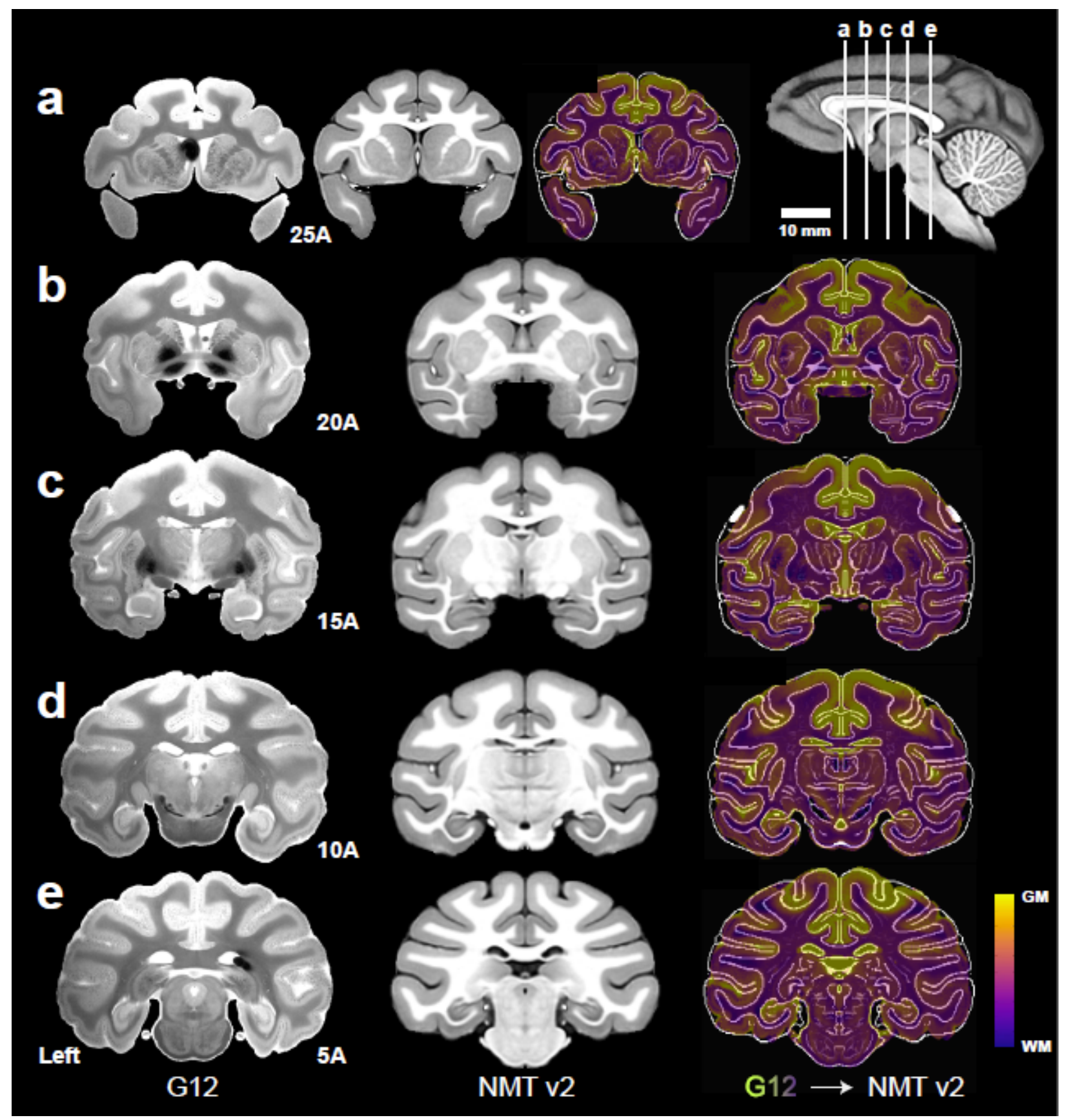

Figure 1. Alignment of subject G12 to the symmetric NMT v2. Panels (a-e) depict coronal slices through the G12 anatomical scan (left), the symmetric NMT v2 (middle) in stereotaxic space, and the nonlinear registration of the $\mathrm{G} 12$ to the symmetric template (right). Slice positions are in $\mathrm{mm}$ anterior to the origin (EBZ; ear bar zero) and are depicted on the midsagittal NMT v2 cross-section (upper right). Parameters for the ANTs registration pipeline were customized to prioritize alignment of subcortical regions. Color ('plasma') shows the warped G12 tissue intensities superimposed on the salient edges of the NMT v2. The darkest purple represents white matter (WM), whereas lighter purple and greens represent gray matter (GM). Left hemisphere depicted on the left side. 
The G12 scan and its subcortical labels were resampled to match the NMT v2 resolution $\left(0.25 \mathrm{~mm}^{3}\right.$ isotropic), and the out-of-plane detail in the G12 was interpolated to match the NMT v2 resolution. Differences in morphology due to their preparations were noted (i.e., sulcal positioning, ventricle size and ex vivo fixation effects, as well as the presence of artifacts like air bubbles), because these may result in nonlinear registration errors or require repositioning greater than what is allowed by the nonlinear registration algorithm (cost function tradeoff). the region edges. These were mitigated with modal smoothing, clustering and outlier detection to make for more natural, locally consistent regions. At this stage, the regions underwent manual correction, followed by additional post-processing (see Section 2.1.4). The 3D consistency of the completed ROls was verified by visualizing the surface of each region using AMIRA and AFNI's surface viewer SUMA (Saad et al., 2004) (Fig. 2). The primary subcortical parcellation that forms the finest hierarchical level of the SARM (level 6; see Section 3.1.2) is

\section{shown in Figure 3.}

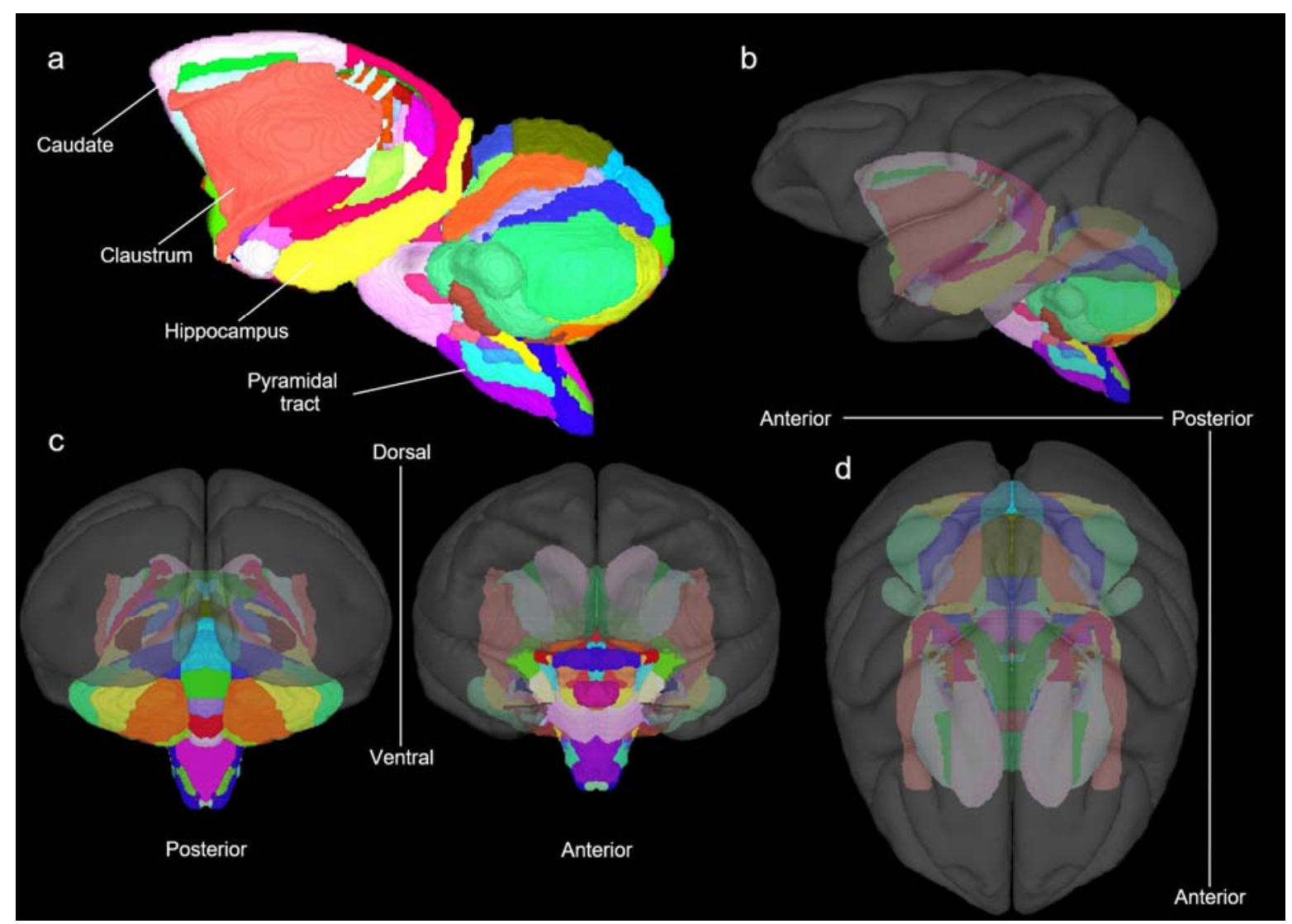
subcortical surfaces, displayed in color using SUMA (Saad et al., 2004). The subcortical regions are 
shown with respect to the NMT v2 surface (shown in gray scale) in a (b) left lateral, (c) posterior (left), anterior (right), and (d) superior view.

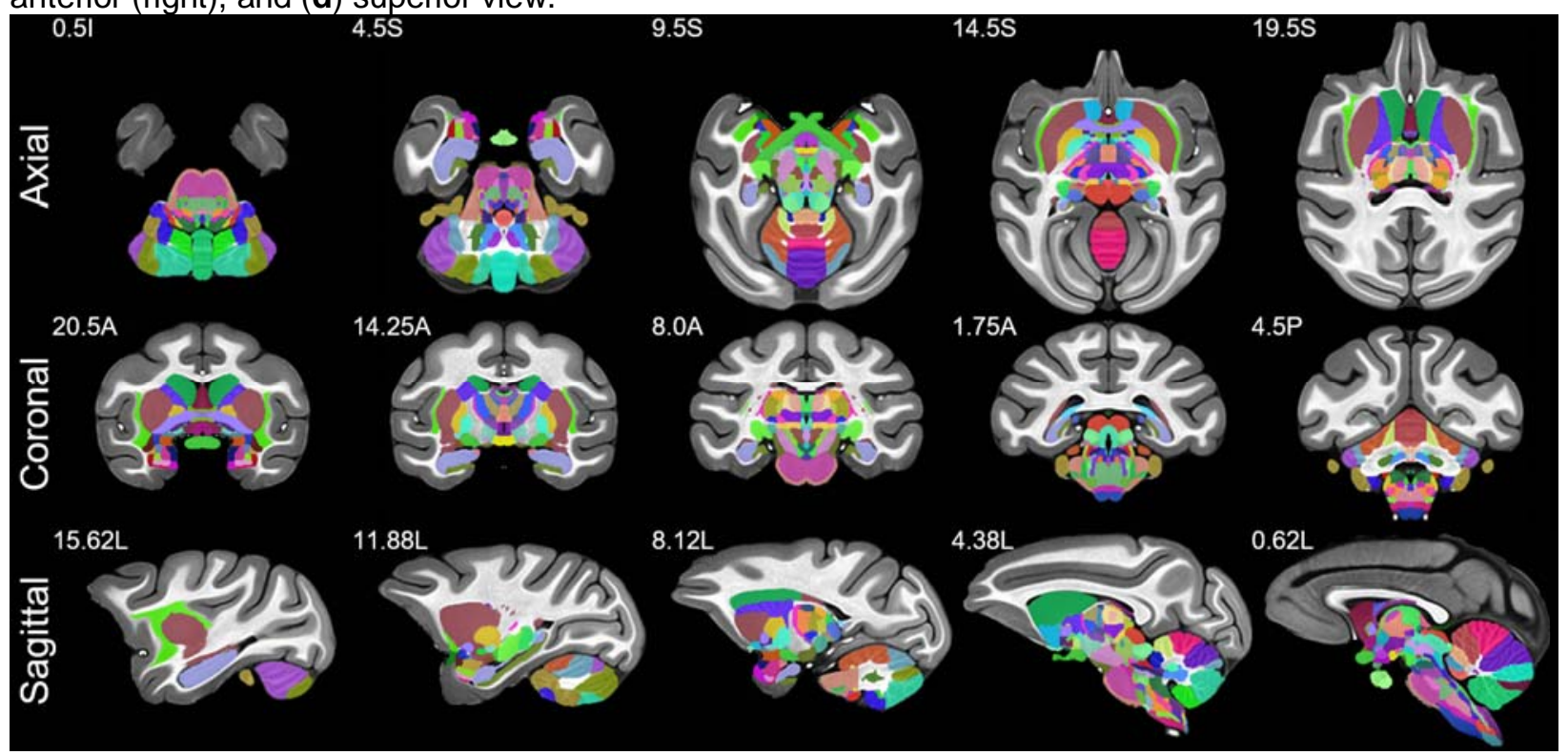

Figure 3. Subcortical regions in NMT v2 space. The subcortical parcellation of the G12 subject was warped to the NMT v2 symmetric template and manually adjusted to match the template's morphology. These regions constitute level 6 of the SARM and are shown in color on the symmetric brain-extracted NMT v2 template. Slice coordinates relative to the origin (EBZ; ear bar zero) are in $\mathrm{mm}$ in the superior/inferior (top), anterior/posterior (middle), and left/right (bottom) directions.

407 The 206 manually-drawn primary ROls comprise the finest level (level 6) of the SARM. 408 Following the same principle as in the CHARM (Jung et al., this issue), these 206 ROls were 409 organized hierarchically into six levels of granularity. Individual ROls were assembled into 410 progressively larger (and, in most cases, spatially contiguous) groups from levels 5 to 1 . Each $411 \mathrm{ROI}$ or group of ROls at a lower level (e.g., level 4) belongs to exactly one group in the next 412 higher level (e.g., level 3). Table 1 shows the number of ROIs in each level and characterizes 413 their volumes in the NMT v2. Whole-brain coronal views of the SARM levels 2, 4, and 6 are 414 shown in Figure 4. The various levels were designed to be suitable for either structural or 415 functional MRI analyses, with their different spatial resolutions. Users can combine more than 416 one grouping level within a single analysis to, for instance, examine the relationships between a 417 specific nucleus and larger composite brain regions. To further illustrate the SARM hierarchy, 418 Figure 5 provides an exploration of the amygdala. The dendrogram demonstrates how the 419 amygdala splits into its constituent regions, and these component structures are depicted on a 420 coronal section for levels 3-6. 
bioRxiv preprint doi: https://doi.org/10.1101/2020.09.16.300053; this version posted September 18, 2020. The copyright holder for this preprint (which was not certified by peer review) is the author/funder, who has granted bioRxiv a license to display the preprint in perpetuity. It is made available under aCC-BY-NC-ND 4.0 International license.

Table 1. Basic characteristics of the SARM hierarchy.

\begin{tabular}{lrrr}
\hline Level & \# of ROls & ROI Vol., median $\left(\mathrm{mm}^{3}\right)$ & ROI Vol., 5-95\% $\left(\mathrm{mm}^{3}\right)$ \\
\hline 1 & 5 & 2,570 & $1,232-8,273$ \\
2 & 15 & 649 & $46-5,228$ \\
3 & 35 & 309 & $55-2,112$ \\
4 & 70 & 123 & $19-1,269$ \\
5 & 167 & 36 & $3-510$ \\
6 & 206 & 36 & $3-497$
\end{tabular}

Table 1. The SARM Group Hierarchy. For each level of the hierarchy, the number of ROls used to parcellate the subcortex, their median volume, and the $5^{\text {th }}-95^{\text {th }}$ percentile of their volumes are listed. At lower levels, ROls are combined into fewer and larger composite structures. The full table of SARM region names, abbreviations, and constituents is provided as a CSV file in the distribution package and is also shown in Table S1.

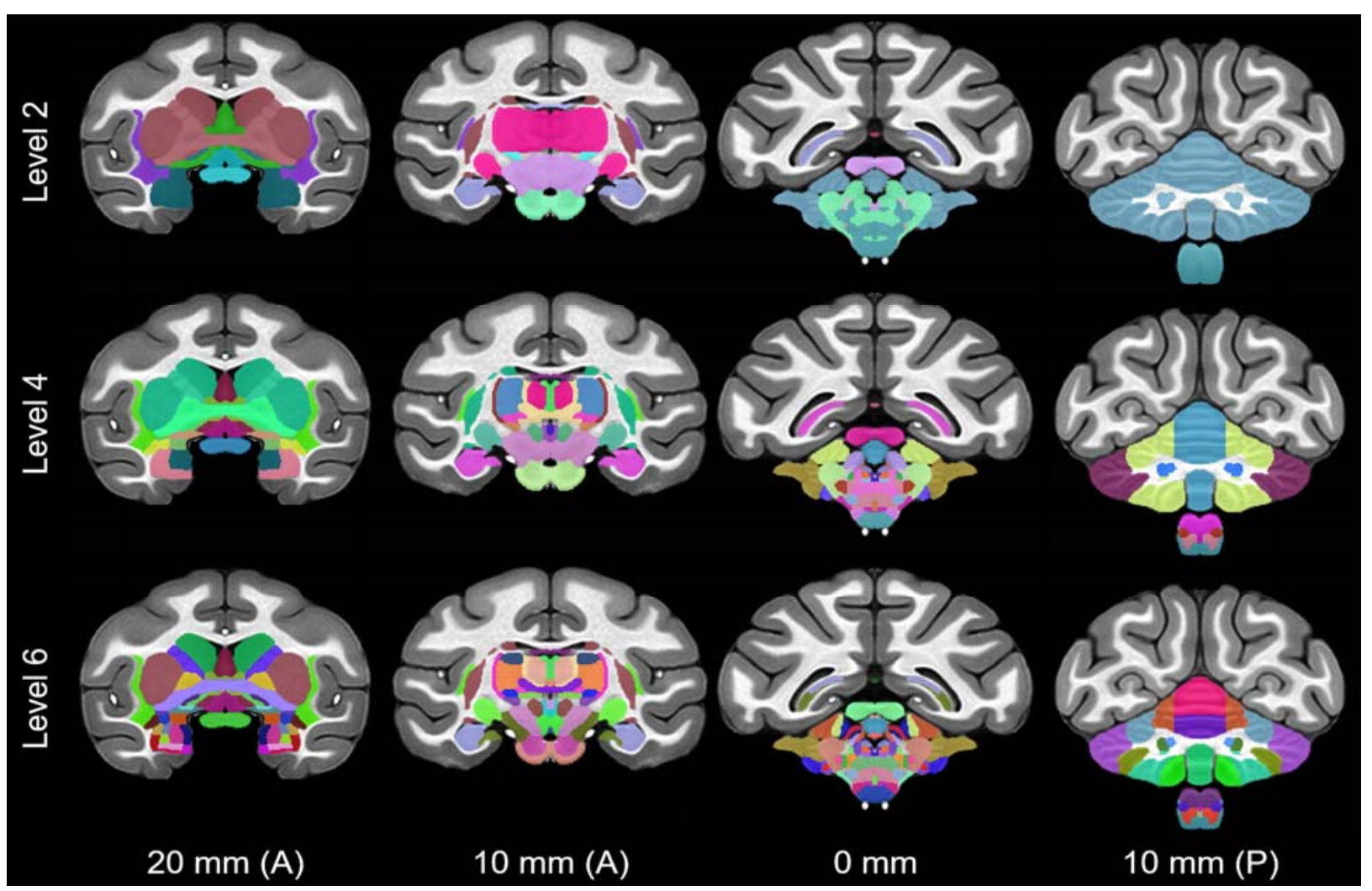

Figure 4. SARM's Hierarchical ROI Groupings. Representative coronal slices through the symmetric

NMT v2 in stereotaxic space, showing levels 2, 4 and 6 of the SARM hierarchy. Level 2 contains relatively broad composite structures, level 4 contains somewhat finer groupings, and level 6 consists of the finest 

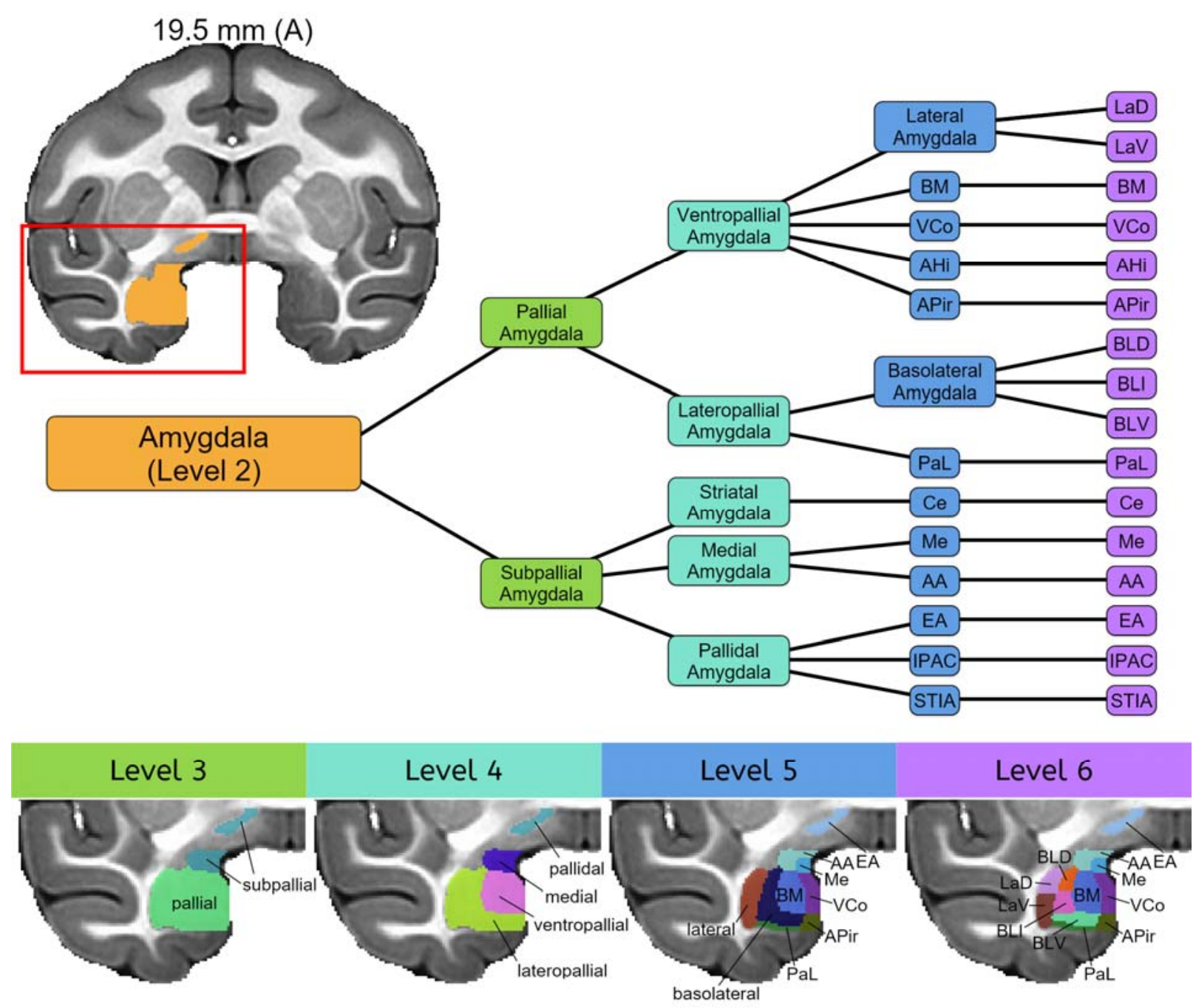

Figure 5. Hierarchical parcellation of the amygdala. The amygdala, a subcortical region within the telencephalon, is shown in orange on the right hemisphere of a coronal section. The bottom row shows amygdala subdivisions for levels 3-6 of the hierarchical atlas in various colors on close ups of the right temporal lobe region contained in the red box on the full coronal section. The color-coded dendrogram shows the hierarchical relationship between the components of levels 3-6. Level 3 distinguishes the portions of amygdala deriving from the pallium and subpallium during development. At level 4, regions arising from particular domains within the pallium or subpallium are differentiated. These regions are further divided into the various amygdala nuclei and subnuclei in levels 5 and 6 . Note other subcortical regions are not shown. Abbreviations: $\boldsymbol{A A}$, anterior amygdaloid area; $\boldsymbol{A H i}$, amygdalohippocampal area; $\boldsymbol{A P i r}$, amygdalopiriform transition; $\boldsymbol{B L D}, \boldsymbol{B L I}$, and $\boldsymbol{B L V}$, basolateral dorsal, intermediate, and ventral amygdaloid n.; $\boldsymbol{B M}$, basomedial amygdaloid n.; $\boldsymbol{C e}$, central amygdaloid n.; $\boldsymbol{E A}$, extended amygdala; IPAC, posterior interstitial nucleus; $L a D$ and $L a V$, lateral dorsal and ventral amygdaloid $n$.; Me, median amygdaloid n.; PaL, paralaminar amygdaloid n.; STIA, intraamygdaloid division of the bed $\mathrm{n}$. of the stria terminalis; VCo, ventral cortical amygdaloid n.. 


\subsubsection{ROI and Hierarchical Grouping Definition}

456 Supplementary Table 1 (Table S1) itemizes the 206 ROls at level 6 and shows their progressive 457 hierarchical grouping, from level 5 to level 1. Level 1 assembles all ROls according to the 458 classical developmental division of the neuraxis, namely the (subcortical) telencephalon, 459 diencephalon, mesencephalon, metencephalon, and myelencephalon. The ordering of these 460 ROls reflects that, together, the subcortical telencephalon and diencephalon comprise the 461 subcortical forebrain, the mesencephalon is synonymous with the midbrain, and the 462 metencephalon and myelencephalon make up the hindbrain. Level 2 divides the telencephalon 463 into lateral and ventral pallium (LVPal), medial pallium (MPal), amygdala (Amy), basal ganglia 464 (BG), diagonal subpallium (DSP), and preoptic (preoptic) regions. The order in which these 465 divisions are listed roughly follows the developmental partition proposed by Puelles et al. 466 (2013), with entirely pallial groups (LVPal and MPal) first, followed by the amygdala with its 467 pallial and subpallial components (see level 3), and, finally, by entirely subpallial groups (BG, 468 DSP and preoptic). At level 2, the diencephalon is divided into the hypothalamus (Hy), 469 prethalamus (PreThal), thalamus (Thal), and epithalamus (EpiThal). The mesencephalon was 470 not divided at level 2, but the region was relabeled "midbrain" (Mid) to match the more common 471 choice of terminology employed beyond level 2. Still at level 2, the metencephalon was split into 472 the pons (Pons) and the cerebellum ( $\mathrm{Cb})$, whereas the myelencephalon remained whole, but 473 switched names to the term medulla (Med). Levels 3 to 6 propose a progressively more refined 474 parcellation of the larger groups of level 2, ending with level 6, which lists each individually 475 drawn ROI. Levels 5 and 6 were left largely similar to allow for future versions of the SARM 476 (now SARM v1) to incorporate additional sub-structures. In general, beyond level 2, the 477 hypothalamic, thalamic, mes-, met- and myel-encephalic ROls were not grouped according to 478 the ontological plan because most of the small ontologically related ROls of these regions are 479 spatially non-contiguous in the adult brain. Instead, these ROls were mainly grouped according 480 to either functional or purely topological criteria, with the practical condition that they remain 481 contiguous, as this has greater relevance for targeting of subcortical regions and neuroimaging 482 analytical strategies (e.g. clustering). The next sections briefly describe the rationale for the 483 drawing of the ROls and their grouping at and below level 2.

484 3.1.3.1 Lateral and Ventral Pallium. The lateral and ventral pallium (LVPal) group contains 4 485 primary ROIs (Table S1). The claustrum $(\mathrm{Cl})$ and the dorsal and ventral endopiriform claustrum 
(DEn and VEn) were all identifiable in the G12 (not shown) and the NMT v2 (Fig. 6a,b). DEn appeared as a separate entity at the 'heel' of $\mathrm{Cl}$ (see arrows in Fig. 6a). VEn was recognized by a consistently lighter contrast in comparison with the darker nuclei of the amygdala (see blue asterisk in Fig. 6a). The small bundle of capillaries (base of the lenticulostriatal arteries) located at the base of the putamen $(\mathrm{Pu})$ and above the 'heel' of $\mathrm{Cl}$ was incorporated into the $\mathrm{Pu} \mathrm{ROI}$ by default (yellow asterisk in Fig. 6a). The piriform cortex (Pir; not shown) was recognized by its thinner cortical width at the medial junction of the orbitofrontal and temporal cortices (Carmichael and Price, 1994; Evrard et al., 2014), although its exact border with $\mathrm{Cl}$ at the limen insulae and with the amygdalo-piriform transition (APir) in the temporal lobe could not be ascertained.

At level 4, DEn and VEn are grouped in En (Table S1). At level 3, En is grouped with Pir in the ventral pallium (VPal), from which En and Pir originate along with other olfactory structures (Puelles et al., 2013) that were not segmented here (but see CHARM; Jung et al., this issue). Also at level 3, $\mathrm{Cl}$ constitutes the only $\mathrm{ROI}$ of the lateral pallium (LPal). At level 2, LPal
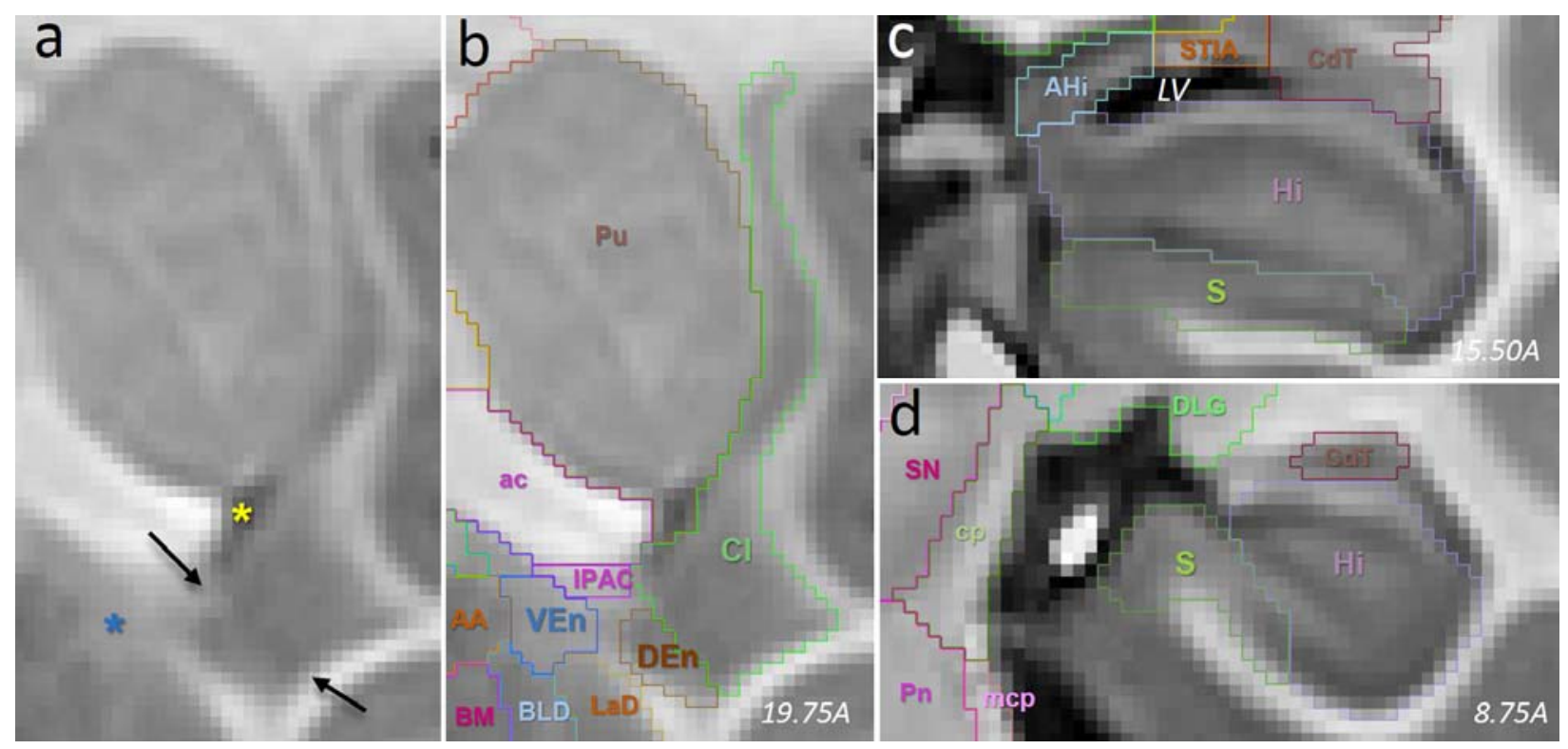

Figure 6. SARM lateral, ventral, and medial pallial ROIs. (a,b) Coronal section through the right hemisphere of NMT v2 (corresp. to RMBSC Fig. 48) with delineations of $\mathrm{Cl}$, DEn and VEn. In (a), the black arrows indicate the border between $\mathrm{Cl}$ and $\mathrm{DEn}$; the blue asterisk marks the consistently lighter contrast of VEn; the yellow asterisk indicates a bundle of capillaries incorporated into the Pu ROI. (c,d) Coronal sections through the right hemisphere of NMT v2 (RMBSC4 Fig. 63 and 77, respectively) illustrating the delineations of $\mathrm{Hi}$ and $\mathrm{S}$. Abbreviations: $\boldsymbol{A A}$, anterior amygdaloid area; ac, anterior commissure; $\boldsymbol{A H i}$, amygdalohippocampal area; $\boldsymbol{C d T}$, tail of the caudate; $\boldsymbol{B M}$, basomedial amygdaloid n.; $\boldsymbol{B} L \boldsymbol{D}$, basolateral amygdaloid n.; $\boldsymbol{c p}$, cerebral peduncle; $\boldsymbol{C l}$, claustrum; $\boldsymbol{D E n}$, dorsal endopiriform claustrum; DLG, dorsal lateral geniculate; Hi, hippocampus; IPAC, interstitial nucleus of the posterior part of the anterior commissure; $\boldsymbol{L V}$, lateral ventricle; $\boldsymbol{m c p}$, medial cerebellar peduncle; $\boldsymbol{P n}$, pontine nucleus; $\boldsymbol{P u}$, putamen; $\boldsymbol{S}$, subicular complex; $\mathbf{S N}$, substantia nigra; $\mathbf{S T I A}$, intraamygdaloid stria terminalis; VEn, ventral endopiriform claustrum. In all panels, left is medial and top is dorsal. 
and VPal are grouped under LVPal.

3.1.3.2 Medial Pallium (Hippocampal Formation). The hippocampus ( $\mathrm{Hi}$ ) and subicular complex (S) were segmented without distinguishing their internal subdivisions (i.e., CA1-3, dentate gyrus, parasubiculum, presubiculum, subiculum per se, and prosubiculum) (Table S1; Fig. 6c,d). They were grouped together, along with the fimbria (fi), as hippocampal formation (HF) at levels 3 and 4. Although being acellular, the fimbria was added to the HF group in order to take into account the lower spatial resolution of functional scans that may not distinguish fi from $\mathrm{Hi}$ and $\mathrm{S}$. The fornix (f) was drawn throughout the lateral ventricle mainly for illustrative purposes. It was not added to the HF group to avoid false attribution of activation possibly originating from regions located in the vicinity of the distant $f$ (e.g., septum and dorsal thalamus). Finally, at level 2, HF and f were grouped together in the medial pallium (MPal), from which they originate (Puelles et al., 2013).

3.1.3.3 Amygdala. The amygdala was delineated into 16 primary ROls (Table S1). Figure 7 514 illustrates the segmentation of the amygdala at one representative anteroposterior level in NMT 515 v2, G12 and RMBSC4. Throughout the anteroposterior extent of the amygdala, the dorsal and 516 ventral parts of the lateral amygdaloid nucleus ( $\mathrm{LaD}$ and $\mathrm{LaV}$ ) were recognizable by their darker 517 contrast, compared to the lighter dorsal, intermediate and ventral parts of the basolateral 518 nucleus (BLD, BLI and BLV). The theoretical location of the basomedial nucleus (BM) often 519 contained a darker region in both NMT v2 and G12, which likely corresponds to the parvocellular or magnocellular division of BM and contrasts with the lighter and more homogeneous ventral cortical nucleus (VCo). The paralaminar nucleus (PaL) appeared as a thin sheet of lighter (G12), and somewhat darker (NMT v2), contrast at the base of the amygdala. The central nucleus $(\mathrm{Ce})$ was less distinct, but its theoretical anatomical location largely corresponded to a circular area with a lighter contrast in NMT v2. Medial to Ce, AA and the medial nucleus (Me) appeared darker in NMT v2 and lighter in G12. The boundaries between Me and AA, between BLD, BLI, and BLV, and between LaD and LaV were drawn based on their theoretical topological localization (Amaral et al., 1992; Stefanacci et al., 2000; Paxinos et al., 2009). Lateral to the amygdala, the amygdalostriatal transition area (ASt) appears as a distinct region, separated by a thin but distinct lighter (NMT v2) or darker (G12) strip of white matter.

530 Dorsal to the amygdala proper, the interstitial nucleus of the posterior part of the anterior 531 commissure (IPAC), the intra-amygdaloid division of the bed nucleus of the stria terminalis 
532 (STIA), and the extended amygdala (EA) are all readily distinguishable in NMT v2. For example,

533 EA appears as a distinct lighter band underneath the ventral pallidum (VP) (Fig. 8a).

As shown in Fig. 5, the amygdala is divided at level 3 on developmental grounds into its pallial and subpallial portions. At level 4, the former splits into the portions arising from the ventral and lateral pallium, while the latter divides into the striatal, medial, and pallidal amygdala. These are then further divided into individual nuclei (level 5) and, in the case of the lateral and basolateral nuclei, into subnuclei (level 6). The different ROls of the pallidal amygdala are not all contiguous. For example, EA has no boundary with other amygdaloid nuclei. Therefore, the pallidal amygdala ROI is one of the two SARM group ROls containing non-contiguous ROls. See 3.1.3.11 for the other exception in the medulla.

3.1.3.4 Basal Ganglia. The basal ganglia (BG) was delineated into 12 primary ROls (Table S1).

543 The head of the caudate $(\mathrm{CdH})$ and the putamen $(\mathrm{Pu})$ were identifiable due to their prominent

544 size and distinct boundary with the internal capsule (ic), anterior capsule (ac), corpus callosum

545 (cc), external capsule (ec), and white matter of the cerebral cortex (Fig. 8). The ventral

546 boundary between $\mathrm{CdH}$ and the anterior portion of the bed nucleus of the stria terminalis (ST)

547 was marked by an abrupt darkening of the signal in ST (see also Section 3.1.3.5). The ventral

548 boundaries of $\mathrm{CdH}$ and $\mathrm{Pu}$ with the accumbens nucleus (Acb; not shown) were identified by a 549 consistent change to a more heterogeneous contrast pattern in Acb. The tail of the caudate 550 (CdT) was distinct all along the lateral ventricle (LV) (Fig. 10) and in proximity to the amygdala, 551 where it borders the amygdalostriatal transition area (ASt; not shown). The external (EGP; Fig. 552 8) and internal (IGP; not shown) globus pallidus were readily identifiable due to their slightly 553 darker contrast, compared to the surrounding white matter. They were distinguishable from one 554 another due to their characteristic shapes and their separation by the thin medial medullary 555 lamina (not shown). The ventral pallidum (VP) appeared distinctly darker between ac and EA 556 (Fig. 8).

At level 5, CdH and CdT were grouped as caudate (Cd), and EGP, IGP and their ventral 558 ansa lenticularis tract (al; not shown) were grouped as globus pallidus (GP). At level 4, Cd, Pu, 559 ASt, and ic (which contains strands of neurons) were grouped as dorsal striatum (DStr), and 560 Acb and Tu were grouped as ventral striatum (VStr). At level 3, DStr and VStr were grouped as 561 striatum. GP, ac and VP were grouped as pallidum (Pd) at levels 4 and 3 . The inclusion of white 562 matter tracts (e.g., ac in Pd) enables using broad ROls in fMRI analyses with rather low spatial 
bioRxiv preprint doi: https://doi.org/10.1101/2020.09.16.300053; this version posted September 18, 2020. The copyright holder for this preprint (which was not certified by peer review) is the author/funder, who has granted bioRxiv a license to display the preprint in perpetuity. It is made available under aCC-BY-NC-ND 4.0 International license.

563 resolution, and in which the BOLD signal would likely 'spread' over multiple neighboring 564 structures, without possible distinction between smaller ROIs.

565 
bioRxiv preprint doi: https://doi.org/10.1101/2020.09.16.300053; this version posted September 18, 2020. The copyright holder for this preprint (which was not certified by peer review) is the author/funder, who has granted bioRxiv a license to display the preprint in perpetuity. It is made available under aCC-BY-NC-ND 4.0 International license.
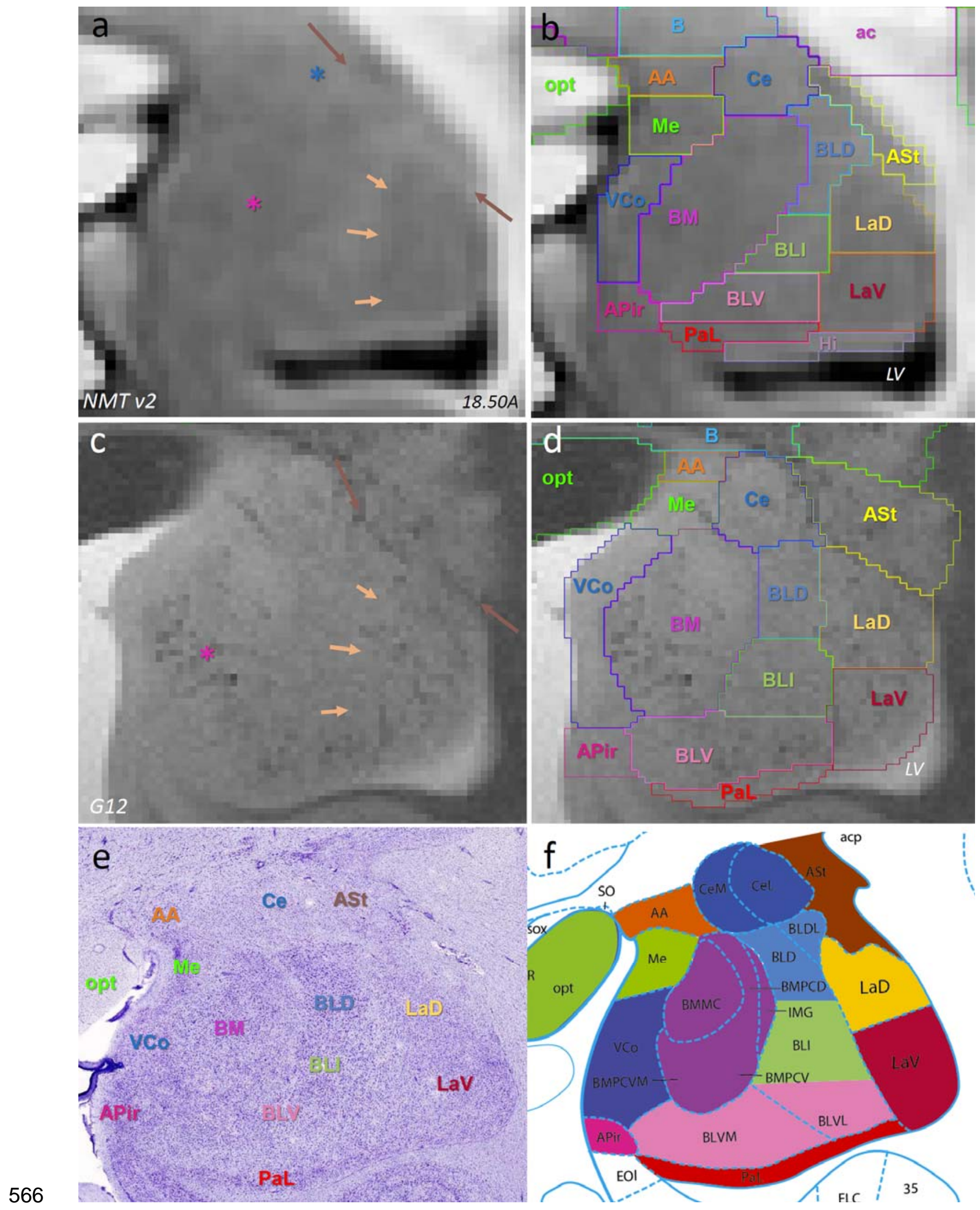

available under aCC-BY-NC-ND 4.0 International license.

Figure 7. SARM's amygdaloid ROIs. Coronal slices through the right hemisphere at approximately the same location in (a, b) NMT v2, (c, d) G12, and (e,f) a corresponding pair of RMBSC4 Nissl-stained section and diagram (Fig. 56). The brown arrow in (a,c) points at the boundary between ASt and the amygdala. The blue asterisk in (a) points at a zone of lighter contrast, likely corresponding to Ce. The light orange arrows in (a, c) point at the putative border of LaD and LaV with BLD, BLI and BLV. The mauve asterisks in panels (a, c) mark the darker contrast included in BM. Abbreviations: $\boldsymbol{A A}$, anterior amygdaloid area; $\boldsymbol{a c}$, anterior commissure; $\boldsymbol{A P i r}$, amygdalopiriform transition area; $\boldsymbol{A S t}$, amygdalostriatal transition area; $\boldsymbol{B}$, basal $\mathrm{n}$.; $\boldsymbol{B} \boldsymbol{L} \boldsymbol{D}, \boldsymbol{B L I}, \boldsymbol{B} \boldsymbol{L} \boldsymbol{V}$, dorsal, intermediate and ventral parts of the basolateral amygdaloid n.; $\boldsymbol{B M}$, basomedial amygdaloid n.; $\boldsymbol{C e}$, central amygdaloid n.; $\mathbf{L a D}$ and $\mathbf{L a V}$, dorsal and ventral parts of the lateral amygdaloid n.; $L V$, lateral ventricle; Me, medial amygdaloid n.; opt, optic tract/chiasma; PaL, paralaminar amygdaloid n.; $\boldsymbol{V C o}$, ventral cortical amygdaloid $\mathrm{n}$.. In all panels, left is
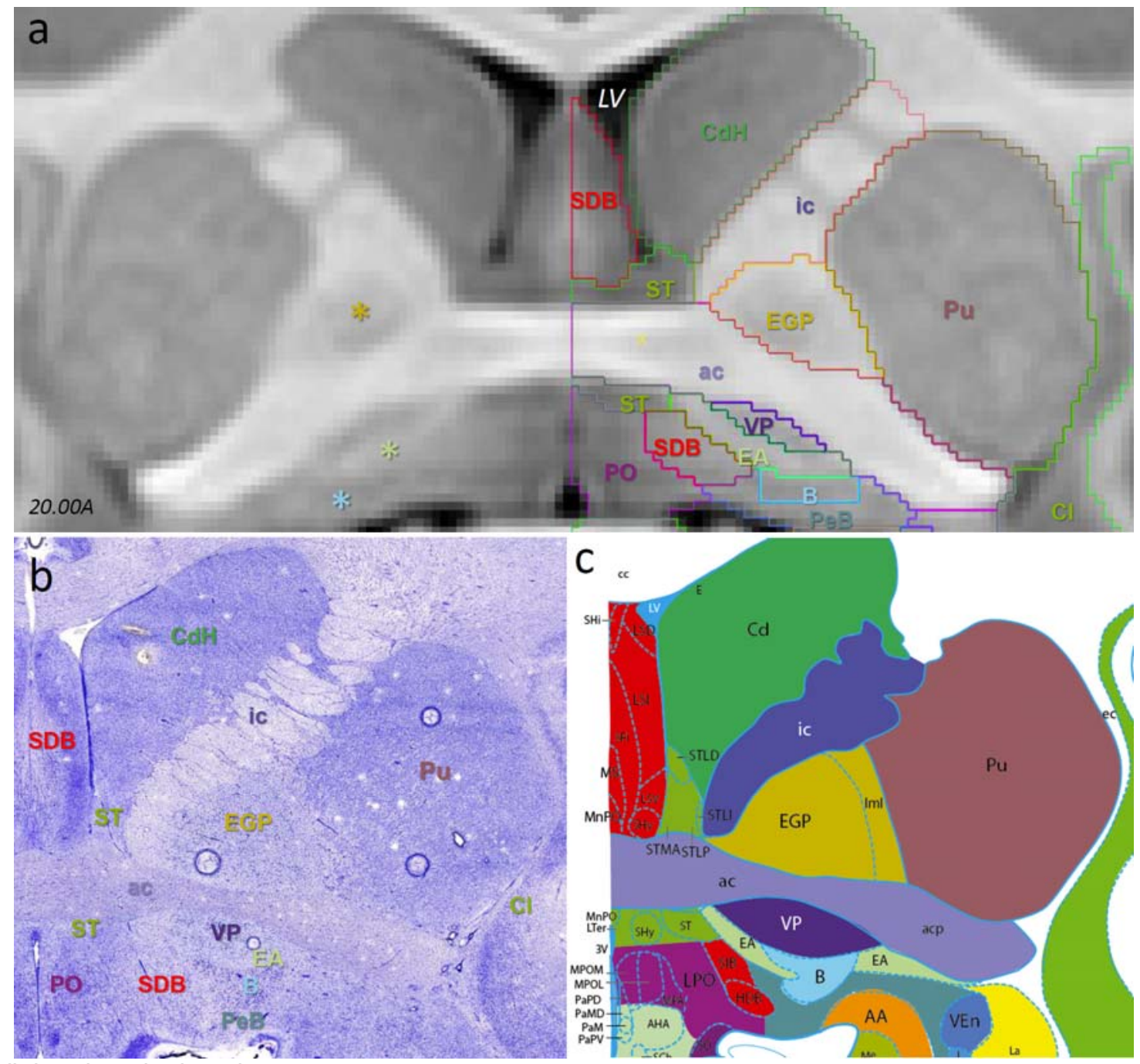

medial and top is dorsal.

Figure 8. SARM telencephalic ROIs. Coronal slices through (a) the symmetric NMT v2 and (b,c) the approximately corresponding pair of RMBSC4 Nissl-stained section and diagram (Fig. 50). The asterisks on the left side in (a) emphasize contrasts corresponding to the location of EGP (yellow), EA (pale green), and $\mathrm{B}$ (blue). Only the anterior portion of SFi is included in the SDB ROI; further caudally, SFi is recognized as a single ROI (see Fig. 9). In all panels, the top is dorsal. In (b,c), left is medial. Abbreviations: $\boldsymbol{B}$, basal nucleus; $\boldsymbol{C d H}$, head of the caudate nucleus; $\boldsymbol{C l}$, claustrum; $\boldsymbol{E A}$, extended

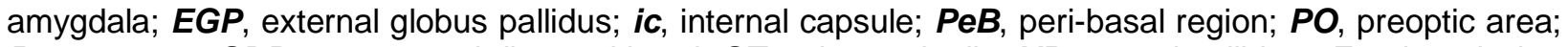
$\boldsymbol{P u}$, putamen; $\boldsymbol{S} \boldsymbol{D B}$, septum and diagonal band; $\boldsymbol{S} \boldsymbol{T}$, stria terminalis; $\boldsymbol{V P}$, ventral pallidum. For the missing 
abbreviations in (c), see Paxinos et al., 2009, where most abbreviations are similar to Paxinos et al., in preparation.

3.1.3.5 Diagonal Subpallium. The ontological definition of the diagonal subpallium (DSP) includes the basal nucleus of Meynert (B), the bed nucleus of the stria terminalis (ST), and different parts of the septum and diagonal band of Broca region (SDB and SFi) (Table S1) (Puelles et al., 2013). B is anatomically formed by an ill-defined group of cholinergic neurons at the base of the basal ganglia. In some slices of NMT v2, the putative location of B could correspond to a slightly darker region ventral to EA and VP (see blue asterisk in Fig. 8a, left); however, this appearance is not consistent. Thus, for the most part, the delineation of $B$ is based on its most likely localization, underneath VP anteriorly (Fig. 8) and in between IGP, the optic tract (opt) and Pu posteriorly (not shown; see for example RMBSC4 Fig. 66). To take into account this less obvious delineation, the region surrounding our delineation of $B$ is labeled as 'peri-basal region' in the SARM v1 (PeB; Fig. 8), which corresponds, in RMBSC4 to a rather undefined region sandwiched between $B$ and other regions such as $A A, C e$ and $S D B$. $B$ and $\mathrm{PeB}$ are grouped in the basal nucleus 'region' (BR) at levels 5 and 4. Anteriorly, the different parts of ST form a distinct 'ring' of dark signal around ac (ST; Fig. 9a,b). Posteriorly, ST mingles with various fiber tracts and appears lighter (Fig. 9a,b). The different components of the medial and lateral septum, as well as those of the diagonal band of Broca, were not readily distinguishable from one another in G12, although the medial portion of the septum appears lighter in NMT v2 and could be ascribed to the medial septum in a future version of the SARM (Fig. 8). Ventrally and posteriorly, SDB (i.e., SIB and HDB in Figure 8c) is consistently darker than EA but lighter than PO, and sits 'sandwiched' between them. For the time being, these anterior and posteroventral regions are grouped in SDB. However, dorsally and posteriorly, one component of the septum that runs along the anterior portion of the lateral ventricle, namely the septimbrial nucleus (SFi), was labeled as a distinct ROI (Fig. 9). SDB and SFi are grouped in

613 the septum diagonal band region (SDBR) at level 4. BR, ST, and SDBR are grouped in DSP at

614 levels 3 and 2. 

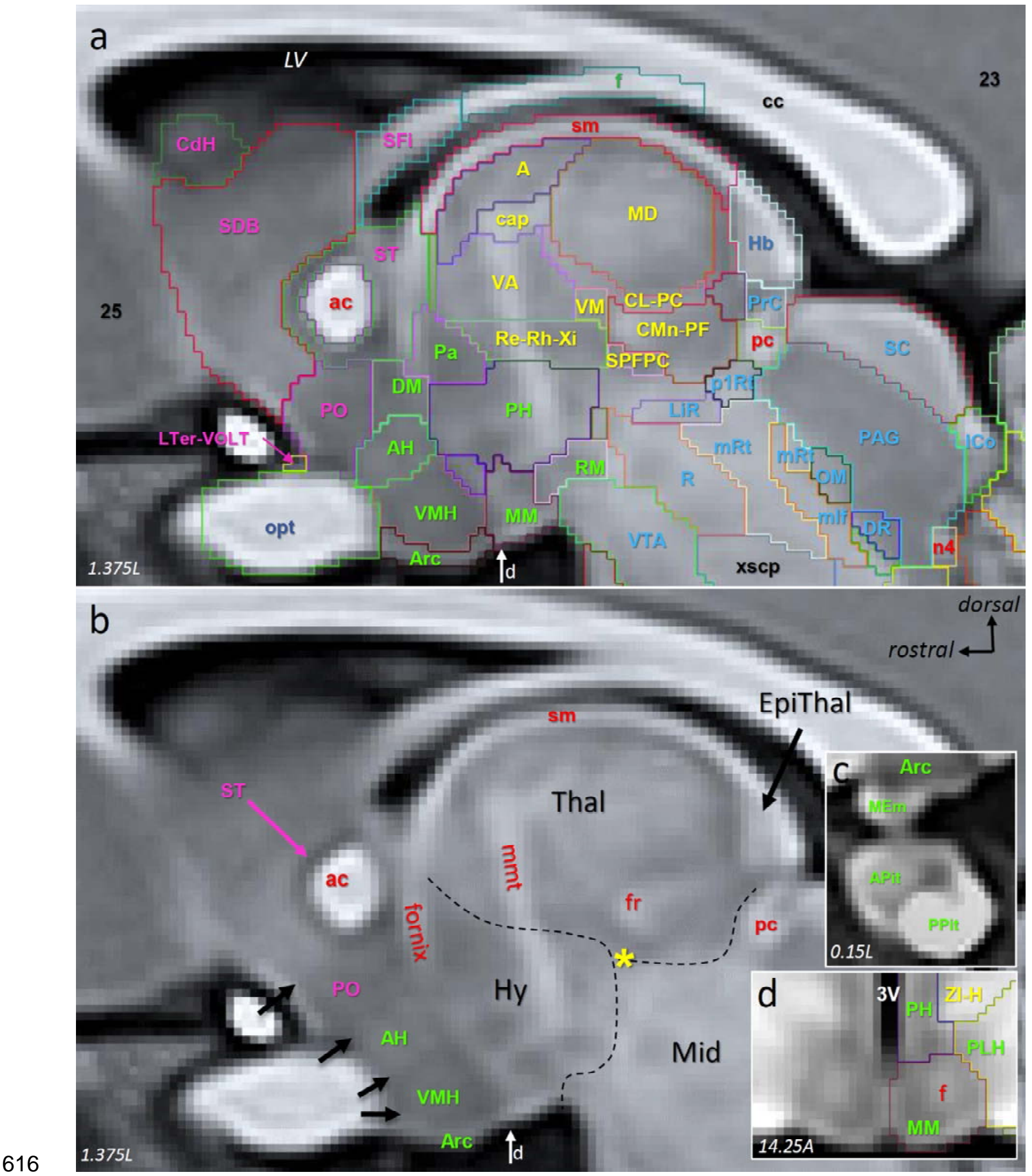

Figure 9. SARM ROIs in parasagittal view. (a, b) Sagittal slice through the NMT v2 showing (a) the delineations of SARM regions and (b) major anatomical landmarks. In (a), ROI labels are color coded: telencephalic (magenta), hypothalamic (green), thalamic (yellow), epithalamic (dark blue), midbrain (light blue), and pons (black). Notable landmarks in (b) include the fr, mmt (not a ROI, inserted in figure for orientation purpose) and fornix. The yellow asterisk is placed just below the distinct darker contrast that 
characterizes SPFPC (see also Fig. 10). The thin dashed lines emphasize the distinctive change in contrast between Thal, Mid, and Hy. The pink arrow points at the ring of dark contrast of ST around ac. The four black arrows on the left side of (b) mark the contrast changes between $\mathrm{PO}, \mathrm{AH}, \mathrm{VMH}$, and Arc. Panel (c) shows a mid-sagittal view of the subjacent (ventral to Arc) pituitary regions APit and PPit, as well as MEm. Panel (d) shows a symmetrical coronal view of MM with a distinctively lighter contrast in its center, corresponding to $\mathrm{f}$. The vertical white arrow at the base of the hypothalamus in panels $(\mathbf{a}, \mathbf{b})$ indicates the anteroposterior level of the coronal view shown in panel (d). Abbreviations: 23 and 25, cortical areas 23 and 25; $\mathbf{3} \boldsymbol{V}$, third ventricle; $\boldsymbol{A}$, anterior thal. n.; ac, anterior commissure; $\boldsymbol{A H}$, anterior hy. n.; APit, anterior pituitary; Arc., arcuate n.; cap, capsule of the anterior thalamic nucleus; $\boldsymbol{c c}$, corpus callosum; $\mathbf{C d H}$, head of the caudate nucleus; CL-PC, centrolateral and paracentral thal. n.; CMn-PF, centromedian and parafascicular thal. n.; DM, dorsomedial hy. n.; DR, dorsal Raphe; EpiThal, epithalamus; $\boldsymbol{f}$, fornix; $\boldsymbol{f r}$, fasciculus retroflexus; $\boldsymbol{H} \boldsymbol{b}$, habenula; $\boldsymbol{H y}$, hypothalamus; $\boldsymbol{I C o}$, inferior colliculus;

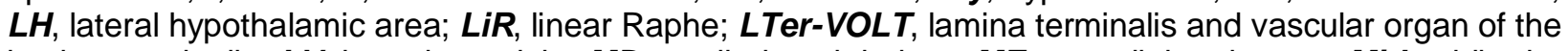
lamina terminalis; $\boldsymbol{L V}$, lateral ventricle; $\boldsymbol{M D}$, mediodorsal thal. n.; $\mathbf{M E m}$, medial eminence, Mid, midbrain; $\boldsymbol{m} \boldsymbol{I f}$, medial longitudinal fascicle; $\boldsymbol{M M}$, mammillary $\mathrm{n}$; $\boldsymbol{m R \boldsymbol { t }}$, midbrain reticulum; $\boldsymbol{n} \mathbf{4}, \mathbf{4}^{\text {th }}$ cranial nerve (crossing); OM, oculomotor complex; p1RT, prosomere 1 reticulum; Pa, paraventricular hy. n.; PAG, periaqueductal gray; $\boldsymbol{p c}$, posterior commissure; $\boldsymbol{P H}$, posterior hy. n.; $\boldsymbol{P L H}$, peduncular lateral hypothalamus; $\boldsymbol{P O}$, preoptic area; $\boldsymbol{P P i t}$, posterior pituitary; $\boldsymbol{P r C}$, precommissural n.; $\boldsymbol{R}$, red n.; $\boldsymbol{R e}-\boldsymbol{R} \boldsymbol{h}-\boldsymbol{X i}$, reuniens, rhomboid and xiphoid thal. n.; $\boldsymbol{R M}$, retro-mammillary n.; $\boldsymbol{S C}$, superior colliculus; $\boldsymbol{S} \boldsymbol{D B}$, septumdiagonal band; SFi, septimbrial n.; sm, stria medullaris; SPFPC, subparafascicular parvocellular thal. n.; $S T$, bed n. of the stria terminalis; Thal, thalamus; VA, ventral anterior thal. n.; VM, ventromedial thal. n.; $\boldsymbol{V M H}$, ventromedial hy. n.; VTA, ventral tegmental area; $\boldsymbol{x \boldsymbol { s }} \boldsymbol{c p}$, crossing of the superior cerebellar peduncle; $\boldsymbol{Z I - H}$, zona incerta and lenticular fascicles ( $\mathrm{H}$ fields).

3.1.3.6 Preoptic Area and Hypothalamus. The preoptic complex (POC; levels 2 and 3 ) and the hypothalamus (Hy; level 2) contain 3 and 17 ROls, respectively (Table S1). At level 4, the POC bifurcates into the preoptic region (POR) and the subjacent segments of the optic nerve and chiasma (opt), which, despite being functionally unrelated, were artificially merged because they frequently 'fuse' at low MRI resolutions. At levels 5 and 6, POR partitions into the different (poorly distinguishable) nuclei of the preoptic area (PO), per se, and the medial lamina terminalis, and its vascular organ (drawn together as LTer-VOLT). LTer-VOLT and opt are readily identifiable throughout G12 and NMT v2, due to their starkly distinct contrast and macroscopic location of LTer-VOLT over opt or at the base of PO (Fig. 9a,b). PO is identified by its canonical location at the level of the optic chiasma and along the anterior part of the third ventricle $(3 \mathrm{~V})$, as well as by its distinct darker contrast. The latter defines rather sharp boundaries with SDB, anteriorly, and with the anterior hypothalamic nucleus $(\mathrm{AH})$, posteriorly, as indicated by the black arrows in Figure $9 \mathrm{~b}$.

Most of the larger subdivisions of Hy are distinguishable due to local variations in signal intensity and/or the presence of specific white matter tracts, such as the mammillothalamic tract (mt). For example, the boundaries between $\mathrm{AH}$, the ventral medial nucleus $(\mathrm{VMH})$, and the arcuate nucleus (Arc) were marked by abrupt changes in contrast, similar to the boundary between $\mathrm{PO}$ and $\mathrm{AH}$ (see black arrows in Fig. 9b). The perifornical (PeF; not shown), retro- 
664 mammillary nucleus (RM) and, more particularly, mammillary nucleus are recognizable by their 665 specific relation to the fornix ( $f$ ) and $\mathrm{mt}$, which are both identifiable as continuous dorsoventral 666 tracts between the thalamus and hypothalamus (Fig. 9b). The mammillary nucleus of the 667 hypothalamus (MM), which typically surrounds $f$, is also recognizable due to the bulge 668 (mammillary body) that it forms at the base of the diencephalon (Fig. 9d). The different divisions 669 of the lateral hypothalamic region (LHy; level 5) - that is, the lateral nucleus (LH), peduncular 670 lateral nucleus (PLH), and juxtaparaventricular nucleus (JPLH) - are delineated mainly based on 671 their lighter signal, compared to neighboring ROls (not shown). The limit between the anterior $672 \mathrm{LH}$ and posterior PLH is set at the level at which the fornix reaches the hypothalamus (not 673 shown; see RMBSC4 Fig. 55). The paraventricular nucleus ( $\mathrm{Pa}$ ) and the posterior hypothalamic 674 nucleus $(\mathrm{PH})$ are distinctively darker and located medially, along 3V. The subthalamic nucleus 675 (STh; not shown; abbreviated elsewhere as STN), which is also part of the hypothalamus 676 (Puelles et al., 2013), is consistently identifiable due to a light circular signal located in-between 677 the darker zona incerta ( $\mathrm{ZI}-\mathrm{H}$; see Section 3.1.3.7) and substantia nigra (SN; see Section 678 3.1.3.8). Finally, the pituitary (Pit; or hypophysis) is connected to Hy via the distinct medial 679 eminence (MEm), and contains an anterior (APit; adenohypophysis) and a posterior (PPit; 680 neurohypophysis) division, which are both recognizable in the NMT v2 due to much brighter 681 contrast for PPit, compared to APit (Fig. 9c).

The hierarchical grouping of $\mathrm{Hy}$ is based mostly on a classical neuroanatomical grouping (Saper, 2012), rather than on ontological grouping (Puelles et al., 2013), due to the non-contiguity of the alar and basal hypothalamic nuclei in the adult Hy. At level 3, Hy is divided into tuberal (THy), posterior (PHy) and pituitary (Pit) groups (Table S1). The tuberal hypothalamus contains the paraventricular hypothalamus ( $\mathrm{Pa}$; also singled out as medial tuberal 687 hypothalamus at level 4), as well as the supraoptic hypothalamus (SOpt), the ventromedial 688 hypothalamic nucleus ( $\mathrm{VMH})$, the medial eminence (MEm) and the arcuate nucleus (Arc), 689 grouped together as ventral tuberal hypothalamus at level 4, and, finally, the anterior 690 hypothalamic area $(\mathrm{AH})$, the dorso-medial hypothalamic nucleus (DM), and the three distinct 691 lateral hypothalamic nuclei ( $\mathrm{LH}, \mathrm{JPLH}$, and PLH), grouped together as dorsal tuberal 692 hypothalamus at level 4 . The level 3 posterior hypothalamus contains the posterior nucleus per 693 se $(\mathrm{PH})$, as well as the prefonical hypothalamus (PeF), the mammillary hypothalamus (MM) and 694 the retro-mammillary hypothalamus (RMM) grouped together as ventral posterior hypothalamus 695 at level 3. Pit forms a separate group at levels 3 and 4, with APit and PPit being considered 696 separately at levels 5 and 6. 
3.1.3.7 Epithalamus, thalamus, and prethalamus. The epithalamus (EpiThal; levels 2-3) contains the pineal gland ( $\mathrm{Pi}$; levels $4-6)$ and the habenula $(\mathrm{Hb}$; levels 4-6). Pi forms a distinct round structure at the midline, above the superior colliculus (SC) (not shown). Hb is located posterior and medial to the thalamus. It is recognizable in NMT v2 by its bright and heterogeneous signal (Fig. 9). The thalamus (Thal) contains $34 \mathrm{ROls}$ at level 6 (Table S1). These ROls remain listed individually at level 5, except for the anterior thalamus $(A)$ and the capsule of the anterior nucleus (cap) (Fig. 9a), which are then grouped to form the anterior thalamus region (AR) ROI. At levels 4 and 3, the ROls are grouped into 12 and 6 larger groups, respectively. At level 4, most of the ROls are grouped based on their connections (e.g., spinal, cerebellar, and palladio-nigral groups) and classical functional attributions (e.g., "non-specific" intralaminar and midline groups). The dorsal lateral geniculate (DLG; abbreviated elsewhere as LGN) and medial geniculate (MG) nuclei remain ungrouped at level 4, due to their size, anatomical distinctiveness, and functional specificity. At level 3, most of the level 4 ROls are grouped into yet larger entities based purely on their location within Thal. MG and DLG are grouped into a geniculate $\mathrm{ROI}$ (GThal). The reticular thalamus (Rt) remains ungrouped until level 2 (Thal) due to its anatomical distinctiveness.

Most level 6 ROls of the thalamus are distinguishable in G12 and NMT v2. For example, Figure 10 illustrates the delineation and signal contrast of several distinct thalamic ROls in one coronal slice of NMT v2. The boundary between some ROls, such as the posterodorsal and posteroventral parts of the ventrolateral nucleus (VLPD and VLPV), had to be based on their theoretical location and topological relationships. But, in most cases, there was a consistent shift in contrast at ROI borders, such as at the boundary between VLPV (darker) and the medial and lateral parts of the ventral nucleus (VPM-VPL; see black arrows in the left side of Fig. 10a). The brighter signal of the VPM-VPL ROI is consistent throughout its anteroposterior extent.

The delineations of the inter-mediodorsal nucleus (IMD), mediodorsal nucleus (MD) and centrolateral and paracentral nuclei (CL-PC) ROls were marked by rather sharp changes in signal, from darker in IMD to much lighter in CL-PC. The brighter signal in the medial part of MD likely corresponds to its medial portion (MDM in RMBSC4), which could be added in a future version of SARM. Dorsal to IMD, the stria medullaris tract, paraventricular and paratenial nuclei were grouped into one ROI (PT-PV-sm) due to their small individual sizes. Ventral to CL-PC, the centromedial and parafascicular complex (CMn-PF) is identifiable by its darker contrast, which reveals the typical wing-shaped form of CMn-PF, and by the passage of the fasciculus 
bioRxiv preprint doi: https://doi.org/10.1101/2020.09.16.300053; this version posted September 18, 2020. The copyright holder for this preprint (which was not certified by peer review) is the author/funder, who has granted bioRxiv a license to display the preprint in perpetuity. It is made available under aCC-BY-NC-ND 4.0 International license.

729 retroflexus (fr). $\mathrm{fr}$ is located just ventral to CMn-PF in Figure 10a, but it can be seen crossing 730 CMn-PF in the parasagittal view of NMT v2 in Figure 9a,b and in the coronal view of Figure 11a. 


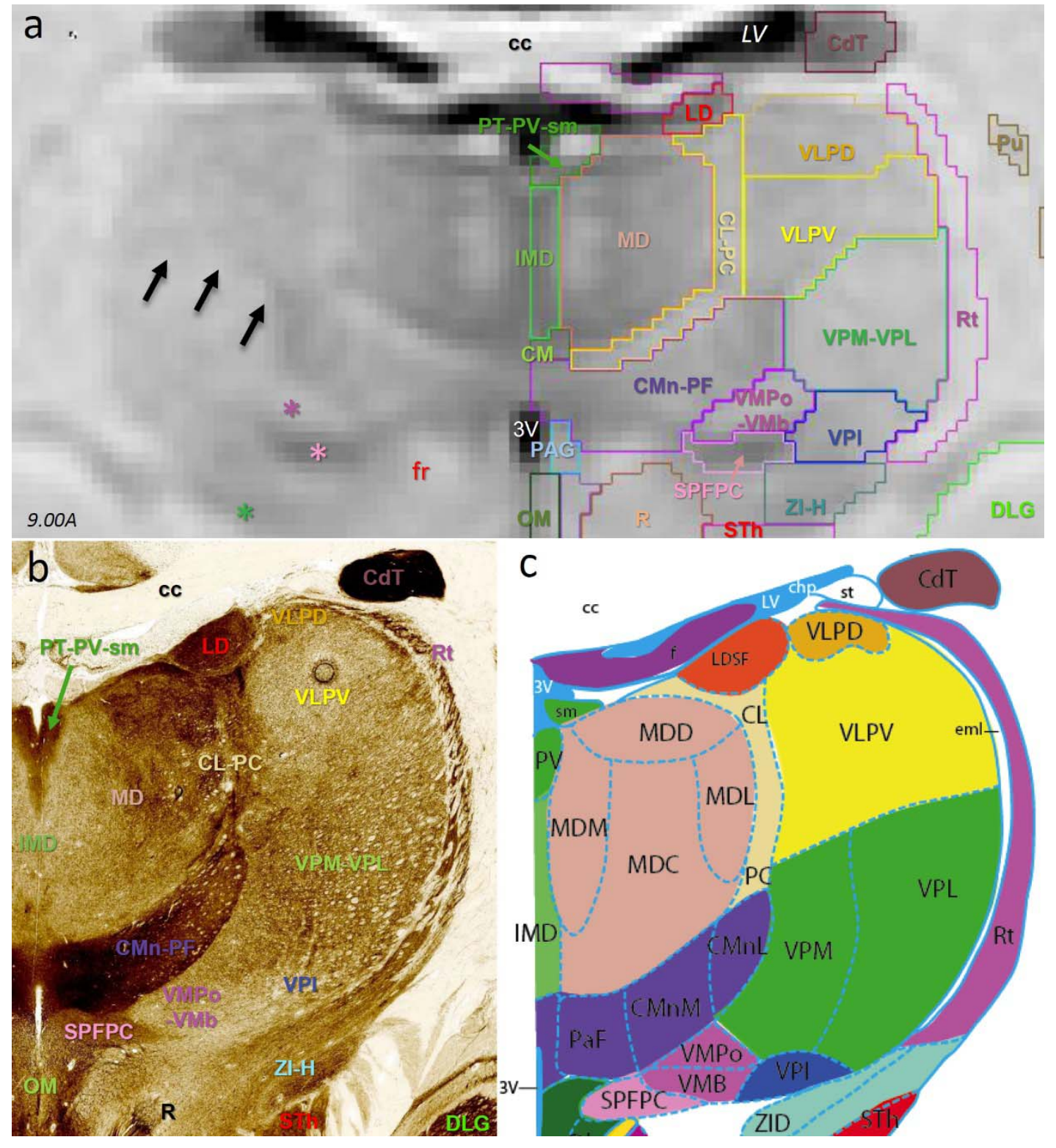

Figure 10. SARM's thalamic ROls in coronal view. (a-c) Coronal slice through the NMT v2 in stereotaxic space (a), and a corresponding pair of RMBSC4 Nissl AChE stain section (b) and diagram (c) (Fig. 71). In (a), the black arrows point at the boundary between VLPV and VPM-VPL. The asterisks emphasize the localizations of VMPo-VMb (dark pink), SPFPC (light pink), and ZI-H (green). The red "fr" indicates the localization of the fasciculus retroflexus, ventral to CMn-PF. (In more posterior slices, fr ascends through CMn-Pf, as illustrated in Fig. 9b and 11a.) Abbreviations (a,b): $\mathbf{3 v}$, third ventricle; CdT, the tail of the caudate; CL-PC, centrolateral and paracentral thal. n.; $\mathbf{C M}$, central medial thal. n.; $\mathbf{C M n}-\boldsymbol{P F}$, centromedial and parafascicular thal. n.; $\mathbf{D L G}$, dorsolateral geniculate thal. n.; IMD, intermediodorsal thal. n.; $\boldsymbol{L D}$, laterodorsal thal. n.; $\boldsymbol{L V}$, lateral ventricle; $\boldsymbol{M D}$, mediodorsal thal. n.; OM, oculomotor complex; PAG, periaqueductal gray; $\boldsymbol{R}$, red n.; $\boldsymbol{R}$, reticular thal. n.; $\boldsymbol{P u}$, putamen; $\boldsymbol{P T}-\boldsymbol{P V}$-sm, ensemble of the stria medullaris, paraventral nucleus and paratenial thal. $\mathrm{n}$.; SPFPC, subparafascicular parvocellular thal. n.; STh, subthalamic n.; VLPD and VLPV, posterodorsal and posteroventral parts of the ventrolateral thal. n.; VMPo-VMB, posterior and basal parts of the ventromedial thal. n.; VPI, ventroposterior inferior thal. n.; VPM-VPL, ventroposterior medial and lateral thal. n.; $\mathbf{Z I - H}$, zona incerta and lenticular fascicles (H fields). For the missing abbreviations in (c), see Paxinos et al., 2009, where most abbreviations are similar to Paxinos et al., in preparation. 
$732 \mathrm{VMb})$ are identified together as a small, brighter region tucked between CMn-PF and the 733 consistently darker and sharply delimited subparafascicular parvocellular ROI (SPFPC; see also 734 Fig. 9a, 10, and 11a). The ventroposterior inferior nucleus (VPI) ROI, which appears lighter in 735 G12 (not shown), is delineated in the NMT v2 mainly based on its theoretical location at the 736 lateral and ventral base of the thalamus, dorsal to the darker ROI of the zona incerta and $\mathrm{H}$ 737 fields $(\mathrm{ZI}-\mathrm{H})$. $\mathrm{ZI}-\mathrm{H}$, which is the only ROI of the pre-thalamus (PreThal), is characterized by a 738 thin strip of darker signal (see green asterisk in the left side of Fig. 10a), sandwiched at more 739 anterior levels between two lighter strips, likely corresponding to the $\mathrm{H} 1$ and $\mathrm{H} 2$ fields of the 740 lenticular fascicle (not present at the AP level shown in Fig. 10). The reticular thalamus ROI (Rt) 741 is defined by a thin lighter 'band' (in coronal slices) covering the lateral aspect of Thal 742 throughout its rostrocaudal extent.

743 3.1.3.8 Pretectum and Midbrain. The small pretectum (PrT) and vast midbrain (Mid) contain 4 744 and 27 ROls, respectively. The posterior commissure (pc) of the pretectum appears distinctly in 745 the sagittal slice in Fig. 9a,b. The other 3 ROls of the pretectum are delineated mainly based on 746 their theoretical location in the vicinity of pc, with, however, a slight contrast differentiation for 747 the precommissural nucleus (PrC, Fig. 9a,b) and, to a lesser extent, the posterior commissural 748 nuclei (PCom-MCPC). The midbrain contains several large and distinct ROls, including the periaqueductal gray (PAG), superior colliculus (SC), inferior colliculus (ICo), and substantia nigra (SN), visible in Figures 9a and 11 (PAG, SC, and ICo). Some smaller ROls could be delineated based on their distinctively darker or lighter contrast (e.g., interpeduncular nucleus,

752 IP; pedunculopontine tegmentum, PTg; caudal pontine reticulum, PnC; dorsal and median 753 Raphe, DR and MR; superior cerebellar peduncle, scp). Finally, other midbrain ROls were 754 drawn based on the localization of the aforementioned distinct ROls. For example, a ventral 755 tegmental ROI was drawn at the base of the midbrain, near its junction with the retro756 mammillary nucleus of the hypothalamus (RM), dorsal to the distinctly darker IP, and in between 757 the ventral halves of SN. Lastly, the red nucleus $(\mathrm{R})$ was drawn based on the occurrence of a 758 slight contrast variation forming an ovoid region, dorsal to VTA (Fig.11a).

In the pretectum, pc and two small adjacent ROls PrC and PCom-MCPC are grouped at level 5 as the posterior commissural region (PCR), to which the prosomeric 1 reticular formation 761 (p1Rt) is added at levels 2-4, to form the PrT ROI. In the midbrain, most level 6 ROls remain the 762 same at level 5, except for the saginum nucleus ROI (Sag-RL), which joins ICo to form the 763 inferior colliculus complex (ICoC). At level 4, ROls are grouped mainly based on coarse 764 anatomical or cytological relatedness. For example, SC and ICoC are grouped into a colliculi 
765 (Co) ROI; the several tegmental nuclei (e.g., microcellular tegmentum, MiTg, and anterior 766 tegmental nucleus, ATg) are grouped into a midbrain tegmentum ROI (TgMid). Similarly, the 767 midbrain dopaminergic complex (DA-Mid) was formed from the large midbrain dopaminergic cell 768 group ROIs (i.e., VTA, SN and RF) and surrounding structures (e.g., IP). At level 3, these ROls 769 are further grouped, mainly based on their cardinal location (i.e., dorsal, lateral, medial, and 770 ventral). 
bioRxiv preprint doi: https://doi org/10.1101/2020.09.16.300053; this version posted September 18, 2020. The copyright holder for this preprint

(which was not certified by peer review) is the author/funder, who has granted bioRxiv a license to display the preprint in perpetuity. It is made available under aCC-BY-NC-ND 4.0 International license.
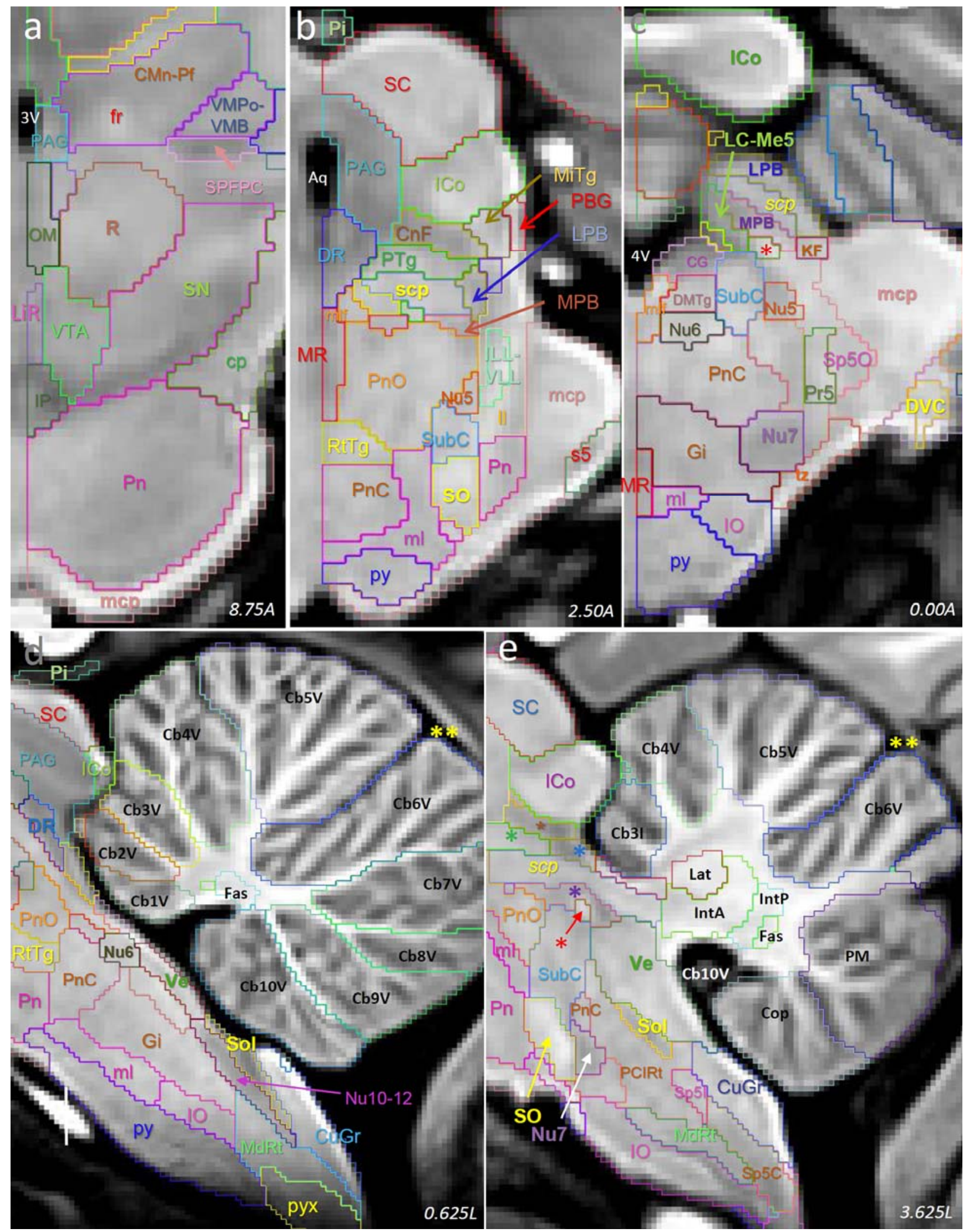

Figure 11. SARM hindbrain ROIs in coronal and parasagittal views. Coronal (a-c) and parasagittal (d,e) slices through the NMT v2 showing ROI delineations at various levels of the neuraxis. In (d,e), the double yellow asterisks indicate the position of the anterior cerebellar fissure that separates anterior and 
posterior lobes. In (e), the asterisks indicate the locations of CnF (brown), PTg (green), LPB (blue), and MPB (purple). The red asterisk indicates the location of the me5. In (a-c), left is medial and top is dorsal. In (d,e) left is rostral and top is dorsal. Abbreviations: $\mathbf{3 V}$, third ventricle; $\mathbf{4 V}$, fourth ventricle; $\boldsymbol{A q}$,

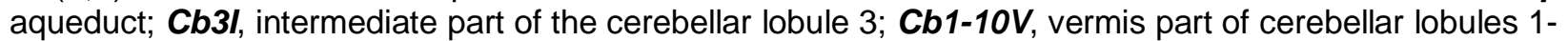
10; CG, central gray n.; CMn-PF, centromedial and parafascicular thal. n.; CnF, cuneiform n.; Cop, cerebellar copula; $\boldsymbol{c p}$, cerebral peduncle; $\boldsymbol{C u G r}$, cuneate and gracile n.; DMTg, dorsomedial tegmentum; $\boldsymbol{D R}$, dorsal Raphe; $\boldsymbol{D V C}$, dorsal and ventral cochlear n.; Fas, fastigial (medial) n.; fr, fasciculus retroflexus; Gi, gigantocellular reticular n.; ICo, inferior colliculus; IntA, anterior interposed n.; IntP, posterior interposed n.; $\boldsymbol{I O}$, inferior olive; $\boldsymbol{K} \boldsymbol{F}$, Kolliker-Fuse n.; Lat, lateral (dentate) n.; $\boldsymbol{L} \boldsymbol{C}$-Me5: locus coeruleus and mesencephalic 5 region; $\mathbf{L i R}$, linear Raphe; II, lateral lemniscus; $\mathbf{P P B}$, lateral parabrachial n.; $\boldsymbol{m c p}$, medial cerebellar peduncle; MdRt, medullary reticular formation; me5, motor trigeminal root; MiTg, microcellular tegmental n.; $\boldsymbol{m l}$, medial lemniscus; $\boldsymbol{m l f}$, medial longitudinal fascicle; MPB, medial parabrachial n.; MR, medial Raphe; Nu5, trigeminal motor n.; Nu6, abducens n.; Nu7, facial n.; Nu10-12, hypoglossal and motor vagus n.; OM, oculomotor complex; PAG, periaqueductal gray; PBG, parabigeminal n.; $\boldsymbol{P C I R t}$, parvicellular and intermediate reticular n.; $\boldsymbol{P i}$, pineal gland; $\boldsymbol{P M}$, paramedian cerebellar lobule; $\boldsymbol{P n}$, pontine n.; PnC, caudal pontine reticulum; PnO, oral pontine reticulum; Pr5, principal trigeminal sensory nucleus; $\boldsymbol{P T g}$, pedunculopontine tegmentum; $\boldsymbol{p y}$, pyramidal tract; $\boldsymbol{p y \boldsymbol { x }}$, pyramidal tract decussation; $\boldsymbol{R}$, red n.; $\boldsymbol{R t T g}$, reticulotegmental formation; $\mathbf{s 5}$, sensory root of the trigeminal nerve; $\mathbf{S C}$, superior colliculus; $\mathbf{s c p}$, superior cerebellar peduncle; $\mathbf{S N}$, substantia nigra; Sol, solitary tract n.; Sp5C, caudal spinal trigeminal n.; Sp5I, intermediate spinal trigeminal n.; Sp50, oral spinal trigeminal nucleus; SPFPC, subparafascicular parvocellular thal. n.; SO, superior olive; SubC, subcoeruleus; $\boldsymbol{t}$, trapezoid bundle region; Ve, vestibular n.; VMPo-VMB, posterior and basal parts of the ventromedial thal. N.; VTA, ventral tegmental area.

3.1.3.9 Pons. The 'pons' region of the metencephalon contains 24 ROls (Table S1), mostly illustrated in Figure 11. The most prominent pons ROI is the pontine nucleus (Pn), located ventrally and well demarcated from the medial cerebellar peduncle (mcp) (Fig. 11a,c,d). The superior olive (SO) forms a bright column posterior to $\mathrm{Pn}$ and directly anterior to the darker Nu7 (Fig. 11b, $c$ and e). The lateral and medial parabrachial nuclei (LPB and MPB) form darker bands around the superior cerebellar peduncle (scp), with LPB being posterior to the lighter PTg (Fig. 11b,c,e). Lateral to MPB, we ascribed a small region to a ROI putatively containing both the locus coeruleus and the mesencephalic trigeminal nucleus (Me5). The localization of this $\mathrm{ROI}$ is supported by the position of the central gray nucleus (CG), recognizable medial to MPB, and the presence of a small lighter region that corresponds most likely to the efferent trigeminal mesencephalic nerve (me5; marked by the red asterisks in Fig. 11c,e). The lateral lemniscus complex $\left(\mathrm{Il}_{+}\right)$, which carries projections from the cochlear nucleus, was identified as a light bundle in the lateral portion of the pons, between SO and ICo, which both receive cochlear inputs. Within the boundaries of $\mathrm{II}_{+}$, we drew ROls most likely to correspond to the position of the dorsal (DLL) and inferior and ventral (ILL-VLL) lateral lemniscal nuclei (Fig. 11b). Other pons regions, such as the oral and caudal pontine reticulum ( $\mathrm{PnO}$ and $\mathrm{PnC}$, Fig. 11b-e), were drawn based on their relative position to the structures that were readily identifiable. 
3.1.3.10 Cerebellum. The cerebellum (level 2) contains 27 ROls (Table S1), including 21 cortical ROls, 4 deep nuclei ROls, and 2 fiber tracts. The 21 cortical areas consist of the 10 lobules, which are partitioned into the more medial vermis lobules (CbV1 to CbV10; Fig. 11d)

819 and four intermediate lobules ( $\mathrm{Cb} 3 \mathrm{I}$ to $\mathrm{Cb} 6 \mathrm{I}$; $\mathrm{Cb} 3 \mathrm{I}$ is shown in Fig. 11e). The intermediate 820 lobules are continuous with and lateral to the corresponding vermis lobules. The other cortical 821 ROls are the paramedian lobule (PM), simple lobule (Sim), copula of the pyramis (Cop), 822 ansiform lobules crus 1 (Crus1) and crus 2 (Crus2) ROls, as well as the floculus (FI) and 823 paraflocculus (PFI). See Figure 11e for PM and Cop. The deep nuclei are the classical lateral or 824 dentate (Lat), anterior interposed (IntA), posterior interposed (IntP), and medial or fastigial (Fas) 825 cerebellar nuclei (Fig. 11e). While the deep nuclei are clearly revealed by an abruptly darker 826 contrast in G12 (not shown), they are identifiable only by a slightly lighter contrast in NMT v2. 827 This slight increase in lightness is, however, sufficient to delineate the edges of each nucleus. 828 The two tracts are the inferior cerebellar peduncle and olivocerebellar tracts, which are both located close enough to the cerebellum to be allocated to this region, instead of others (unlike mcp and scp, which are mostly represented outside the cerebellum).

At level 5, the vermis lobule ROls are grouped into anterior and posterior vermis ROls (AVCbCx and PVCbCx) based on the boundary defined by the primary fissure (double yellow asterisks in Fig. 11d,e) between $\mathrm{Cb5V}$ and Cb6V. In addition, the intermediate lobule ROls, along with Cop, Sim and PM, are grouped into an intermediate cerebellar cortex ROI (ICbCx). Also at level 5, Crus 1 and 2 fuse into a lateral cerebellar cortex (LCbCx) ROI, and FI and PFI fuse into the FI-PFI ROI. At level 4, the vermis cerebellar cortex (VCbCx) ROI combines the anterior and posterior vermis ROls, the deep cerebellar nuclei (DCb) ROI merges the deep nuclei into one, and a cerebellar 'white matter' (wmCb) ROI captures the two fiber tracts. At level 3, all the cortical ROls are grouped under a cerebellar cortex $\mathrm{ROI}(\mathrm{CbCx})$, which then coexists with the DCb and wmCb ROls.

3.1.3.11 Medulla. The myelencephalon (level 1) or medulla (level 2) contains 26 ROls 842 (Supplementary Table 1). Figure 11d,e and 12 illustrate several of these ROls. The most 843 obvious ROls were the solitary tract nucleus (Sol), hypoglossal and motor vagus nuclei (Nu10-

844 12) lying directly ventral to Sol, and the facial motor nucleus (Nu7), due to their sharply delimited 845 darker contrast. The dark Nu7 markedly contrasted against the bright contrast of SO, which lies 846 just anterior to Nu7 (Fig. 11e). The vestibular (Ve) and cuneate-gracile nuclei (CuGr) formed 847 characteristic domes rostral and caudal to Sol, respectively (Fig. 11d). Ventrally, the pyramidal 848 tract (py), decussation of the pyramidal tract (pyx), and inferior olive (IO) were identifiable by 
849 their bulging morphology and heterogeneous contrast (Fig. 11d,e; Fig. 12a,b). The cochlear

850 nuclei (DVC, Fig. 12) formed a distinct structure located lateral to the medulla, within the 851 vestibulocochlear nerve (n8). The oral, intermediate (Fig. 12), and caudal spinal trigeminal 852 nuclei (Sp50, Sp5I, and Sp5C) form a continuous rostrocaudal column made of a medial 853 cellular region (the nucleus itself) and of a lateral fibrous region (the nerve, sp5). Other 854 structures, such as, for example, the paragigantocellular (Gi; Fig. 12) and medullar (MdRt) 855 reticular nuclei, presented a rather homogeneous appearance and were drawn based on their 856 theoretical localization, in between the identifiable regions.

857 At level 5, Sp50, Sp5I, and Sp5C are grouped into a larger spinal trigeminal nucleus 858 ROI (Sp5). At level 4, the ROls are grouped into 8 composite structures based on their 859 functional relatedness. For example, Ve, DVC, n8, and Pr were grouped in a larger vestibulo860 cochlear complex (VCC). Among the 8 composite level 4 ROls, the medullar Raphe 861 (MedRaphe) and medullar motor nuclei (MedMC) are composed of non-contiguous primary 862 ROls. Finally, at level 3, the ROls were grouped based on basic cardinal direction (dorsal, 863 intermediate, and ventral medulla). 


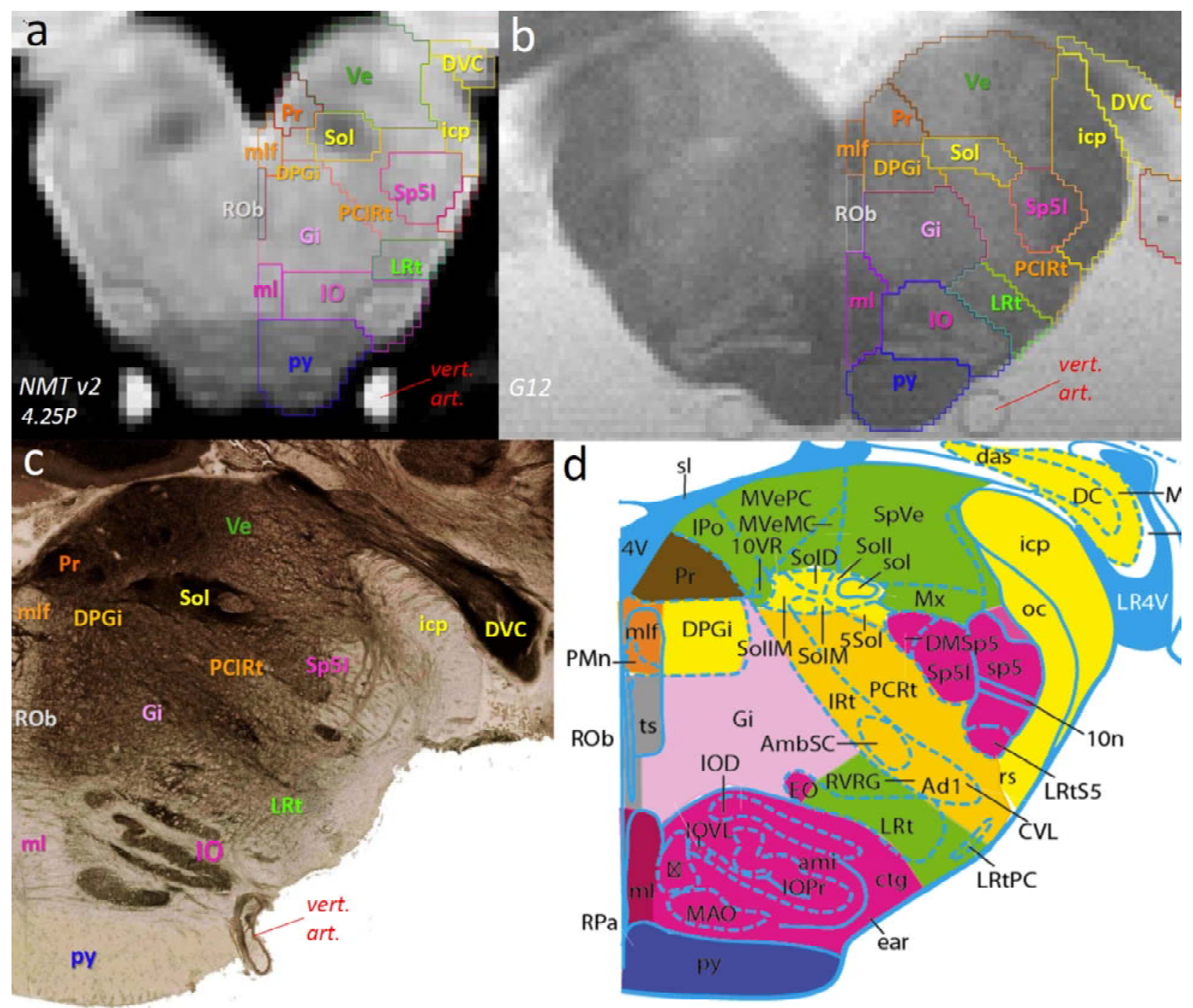

Figure 12. SARM medullar ROIs in coronal view. Coronal slices through the left and right hemispheres of (a) the symmetrical NMT v2 and (b) the G12. Corresponding RMBSC4 slice through the right hemisphere showing (c) an acetylcholinesterase staining and (d) its diagram (Fig. 109). Abbreviations: $\boldsymbol{D V C}$, dorsal and ventral cochlear n.; DPGi, dorsal paragigantocellular nucleus; Gi, gigantocellular reticular $\mathrm{n}$; ; icp, inferior cerebellar peduncle; IO, inferior olive; $\boldsymbol{L R t}$, lateral reticular $\mathrm{n}$;; $\boldsymbol{m I}$, medial lemniscus; mIf, medial longitudinal fascicle; PCIRt, parvicellular and intermediate reticular n.; $\boldsymbol{P r}$, prepositus n.; py, pyramidal tract; $\boldsymbol{R O b}$, Raphe obscurus n.; Sol, solitary tract n.; Sp5I, intermediate spinal trigeminal $n$.; Ve, vestibular $n$. In all panels, left is medial and top is dorsal. For the missing abbreviations in (d), see Paxinos et al., 2009, where most abbreviations are similar to Paxinos et al., in 874 preparation. 


\subsubsection{Individual registration to SARM in NMT v2}

879 The functional localizer data from three rhesus macaque subjects (M1-M3) were nonlinearly 880 registered to the NMT v2 template space for analysis. The quality of the anatomical registration 881 was visually checked (Sections $2.23 \& 2.24$ ). As an illustration of the alignment quality, 882 Supplementary Figure 2 shows the anatomical correspondence between the three macaque 883 subjects and the NMT v2 in the coronal and sagittal planes. Representative coordinates within 884 the periaqueductal gray (PAG, a midbrain region), and the dorsolateral geniculate (DLG) of the 885 right thalamus are labeled.

\subsubsection{Subcortical Activation Clusters}

887 A functional paradigm was used as a validation test to determine whether the SARM could 888 sufficiently localize activity to an expected subcortical region. For this, we used fMRI data 889 collected from three monkeys during a visual stimulus paradigm (flickering checkerboard) that 890 was shown by Logothetis and colleagues (1999) to robustly activate the DLG, which is also 891 known as the lateral geniculate nucleus, or LGN. All functional volumes (i.e. time points) 892 collected were included in the analysis. From this analysis (AFNI- and SPM-based), the 893 statistical results were computed across 2 functional scan sessions per individual. For both 894 analysis packages, we found a consistent, bilateral response within and in the vicinity of the 895 DLG (Fig. 13). To compare the extent of functional activity to the anatomically defined DLG, the 896 significant functional activity correlated with the visual flicker stimulus in monkey subject 3 (M3 897 in Fig. 13a; $p=0.05$, FDR-corrected) is shown in conjunction with the contour of the DLG in the 898 NMT v2 (SARM levels 4-6; Fig. 13b). In the case of subject M3, almost all of the DLG was 899 activated as determined by the fraction of functionally activated voxels in the atlas DLG (Figure 900 13c). BOLD activity within this SARM region was consistently positive in all 3 macaques during 901 presentation of the visual stimulus (Figure 13d). The average BOLD percent signal change 902 across the DLG for all subjects and hemispheres was found to be $0.28 \pm 0.07 \%$ (mean $\pm S T D$ ). To 903 illustrate the extent of functional activation spread, a 3D rendering of subject M3's DLG904 localized activation clusters with the atlas DLG (underlaid) was created using SUMA (Saad et al. 905 2004; Figure 13e). This analysis shows how the SARM can be used for quantifying BOLD 906 activity in individual ROIs and determining the specificity of functional activation. The SARM 


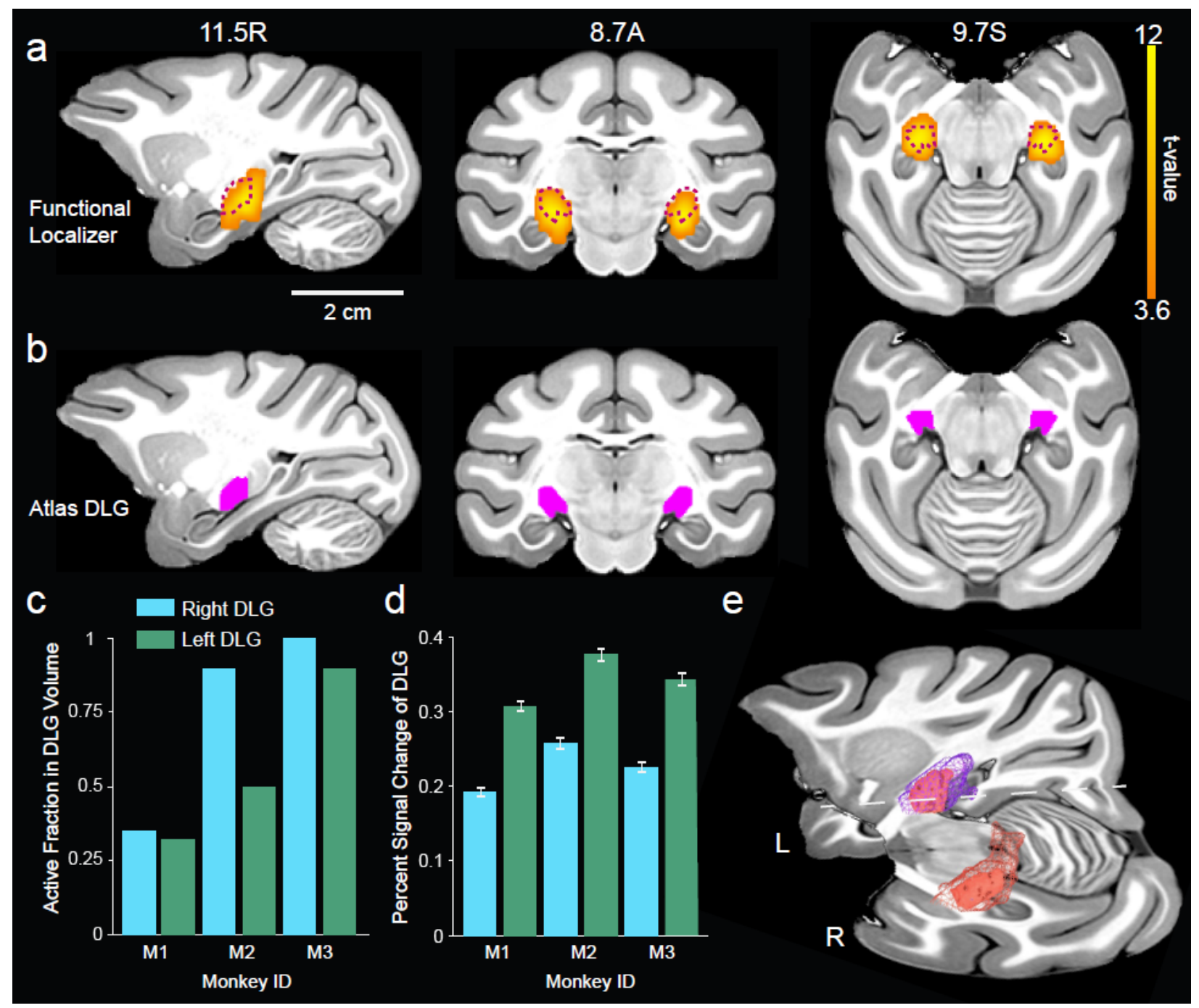

Figure 13. Functional Localizer for DLG. The functional activity elicited in anesthetized monkeys by a flickering checkerboard stimulus was evaluated using the atlas-defined Dorsal Lateral Geniculate (DLG) region. (a) Significant positive BOLD activity elicited in monkey M3 is shown on three sections of the NMT v2 volume that include the DLG. Color shows the t-value of significantly activated voxels (FDR-correction at $p=0.05$; results calculated by the SPM12 analysis pipeline). The anatomical borders of the SARM's DLG are shown in (b) in magenta and with a dashed outline in (a). Slice coordinates are in mm relative to the origin (EBZ; ear bar zero). DLG activation in each hemisphere was quantified for 3 macaque monkeys (Monkey IDs: M1-M3) by (c) the fraction of functional voxels within the DLG region that were significantly activated ( $p<0.05$, FDR-corrected) and (d) the percent signal change (i.e., beta coefficient) associated with the flickering checkerboard (i.e., the $4 \mathrm{sec}$ stimulus $\mathrm{ON}$ period) averaged across all functional voxels in the DLG. Error bars plotted represent the standard deviation. (e) 3D renderings of the DLG as defined by the atlas (smaller) and functionally by the localizer (larger) displayed in SUMA for monkey M3, against an intersecting axial and sagittal slice (unthresholded, $p<0.07$; results calculated by the AFNI analysis pipeline). 


\section{Discussion}

Here, we have introduced the SARM, a digital neuroanatomical parcellation atlas of the macaque monkey subcortex. This atlas is mapped onto the symmetric NMT v2 population template, which reflects the average morphology of an adult rhesus macaque. The SARM offers a subcortical reference matrix that is suitable for the localization of any neuroimaging results in single-subject and group analyses, as well as for experimental surgical planning. Being in fixed stereotaxic coordinates, the SARM referential remains fixed, regardless of changes in border definitions or the nomenclature of anatomical regions with subsequent optimizations. The atlas was originally drawn on the high-resolution coronal sections of an ex vivo MRI of a single subject, with reference to histological material from other subjects, and then manually revised after nonlinear alignment to the in vivo population template. Subcortical areas in the forebrain, midbrain, and hindbrain were parcellated according to the Rhesus Monkey Brain in Stereotaxic Coordinates atlas (Paxinos et al., 2009), with revisions that will be reflected in a new edition (Paxinos et al., in preparation). Not all of the small subcortical cytoarchitectonic regions defined in the RMBSC4 ( 900) were drawn. Instead, we incorporated, in larger ROls, small cytoarchitectonic structures that cannot be identified using the lower MRI resolution, and that would not be pertinent for the localization of BOLD activity. Some of the smaller cytoarchitectonic regions within a single $\mathrm{ROI}$ may be functionally unrelated, as they were grouped 'around' a larger region, mainly based on their spatial proximity. However, Table S1 lists all the small cytoarchitectonic regions assumed to be included within each SARM ROI. This will allow users to evaluate, based on their paradigm, whether the main or a smaller region might be responsible for the observed BOLD signal.

Refinements of the SARM will be released periodically based on user community feedback. In addition, SARM describes the anatomy at 6 different spatial scales, so that it can be used to name and localize small nuclei, mid-size structures suited to describe fMRI activations, and the major developmental divisions of the subcortex. Finally, we tested and validated the SARM using a DLG functional localizer to localize and quantify BOLD activity with 951 respect to different subcortical regions. Further analyses can essentially be computed using the 952 SARM parcellation presented here, whereby functional activity from any atlas label can be 953 assessed both in terms of areal specificity and sensitivity.

954 The utility of an MRI atlas is largely determined by how well data can be warped 955 between the native individual space and the common space of the atlas. Data is commonly 
956 warped to the common space of an atlas for analysis but may also be warped from the common

957 space to an individual scan. Achieving an accurate registration between the source and target

958 datasets is critical for these processes. By providing the SARM on the in vivo population NMT

959 v2, we hope to facilitate simple and accurate alignment between in vivo functional data and a

960 template that closely matches its morphology. To obtain an accurate subcortical atlas, special

961 attention was paid here to ROI positioning after alignment of the G12 parcellation to the NMT

962 v2. We assessed this alignment visually on the structural template and examined various

963 interpolation and regularization schemes to minimize errors. Residual inconsistencies between

964 the subcortical labeling and the NMT v2 structure were manually corrected, ensuring an

965 accurate representation of the subcortex in the NMT v2 space.

As there are many spatial scales by which the macaque subcortex can be subdivided, one strategy is to parcellate different brain areas as finely as afforded by cytoarchitectonics. However, for MRI studies, such a fine parcellation is often unnecessary as the discernible differences between structures are limited by image resolution. Further, the fine scale of some cytoarchitectonic structures can be problematic. Small or thin structures are susceptible to large distortions during nonlinear alignment or resampling and may introduce discontinuities or

972 abnormal shapes or cause a region to disappear entirely. Additionally, after resampling to an

$973 \mathrm{fMRI}$ grid, some small regions might only consist of a few voxels, making averaging over such

974 ROls limited, statistically underpowered, and sensitive to registration errors. To avoid this,

975 individual researchers may combine regions to ensure they are robust and adequately sample

976 the desired area, but this can introduce ambiguity. For instance, one researcher's definition of

977 the regions comprising the amygdala may differ from another, and replicability suffers when

978 researchers lack a consistent definition of brain structures. Atlases circumvent this issue by

979 providing independent, structurally defined regions for ROI analysis and quantification. This

980 capability additionally avoids the potential pitfall of circular analysis, where functional activity is

981 localized to a group of structures, and those structures are then analyzed using the same data

982 (Kriegeskorte et al., 2010).

983 The SARM addresses both the issues of ROI size and consistency by introducing 984 hierarchical groupings. The fine-to-gross classification provides the specificity needed for use 985 with histological material, high-resolution structural scans and targeted brain interventions as 986 well as larger well-defined composite regions suited for reliable fMRI sampling. The SARM's 987 finer levels (levels 5 and 6) may have some utility at typical fMRI resolutions but are rather 988 advantageous for detailed structure analysis (e.g., MRI voxel intensity, comparisons to 
histological material, describing surgical, tracer or pharmacological injection sites). The composite regions of levels 1-4 are sufficiently large to limit the impact of nonlinear registration errors and to include a sufficient number of voxels for averaging over a ROI.

The SARM's composite ROls are additionally useful for meta-analyses and crossspecies comparisons, as finer parcellations differ between anatomists, species (NHP and

994 human), and individuals. By providing composite structures based on cytoarchitectonics and developmental regions, we defined regions that can be used to analyze a target structure at resolutions suited for structural MRI, diffusion MRI and fMRI studies. Conveniently, the SARM can be used in conjunction with the Cortical Hierarchical Atlas of the Rhesus Macaque (CHARM; Jung et al., this issue). This provides researchers with an additional degree of freedom to study subcortical-cortical relationships at varying scales.

There are a few limitations of the current atlas implementation that could be improved in future iterations. First, while the in-plane resolution of the high-resolution scan (G12) was sufficient for definition of subcortical structures, the out-of-plane resolution $(1 \mathrm{~mm})$ limited the available information for tracking these structures in the anteroposterior axis. When these structures were warped to scans with higher out-of-plane resolution, manual adjustments were necessary to resolve discontinuities and inaccurate labeling driven by resampling. Isotropic high-resolution imaging in all dimensions facilitates the creation of digital atlases and their generalizability. Secondly, collecting an in vivo scan of the G12 subject would have helped to account for disparities between in vivo and ex vivo preparations and could have acted as an intermediate target when warping between the high-resolution ex vivo scan and the in vivo NMT v2. Thirdly, in vivo and ex vivo multimodal neuroimaging would have been helpful in delineating fine boundaries of subcortical structures and improved alignment to scans with differing contrast. It may also permit detecting structures that were not readily identifiable here (e.g., Ce).

Another consideration unique to NHP imaging is the orientation of the brain in the scanner. Humans are typically scanned in the supine position. However, macaques are scanned in various positions. The "sphinx" position is the most common, but being seated in a vertical scanner is also fairly common. Visual inspection of scans collected in the sphinx and 1017 seated positions suggest a change in the brainstem's orientation with respect to the rest of the 1018 brain. Affine alignment is unable to correct for such relative differences in brainstem orientation, 1019 and depending on the algorithm, even nonlinear alignment tools may be limited, insofar as the 1020 brainstem can be adjusted. Further investigation is required to evaluate how well brainstem 
1021 structures are registered between a stereotaxic template and functional data collected in the 1022 vertical seated orientation. Additionally, it is worth noting that ex vivo tissue sections may differ 1023 from brain imaging due to differences in features (i.e., brainstem orientation, CSF volume, 1024 ventricle size, and sulcal position). In particular, coronal sectioning through the brainstem for 1025 histological analysis might be perpendicular to the rostrocaudal axis, whereas for an MR scan, 1026 coronal sections are generally oriented with respect to the telencephalon.

Users must carefully consider the issue of alignment between their datasets and the 1028 atlas in template space. If alignment is not done properly, a mislocation in ROI assignment can occur. The same notion applies when attempting to warp an atlas to individual scans. The SARM can always be further improved by using additional isotropic high-resolution structural scans, by using Positron Emission Tomography (PET) with chemoarchitectonically specific radiolabeled ligands (e.g., Oler et al., 2012) or by applying other functional localizers (e.g., mechanoreceptive stimuli for localizing activity in thalamic nuclei CuGR and VPL-VPM or auditory stimuli to activate DCV and MG). The SARM atlas regions can be further organized by functional modalities and connectivity-based clustering. It is strongly recommended to examine the vicinity of functional activations and evaluate the relevance of BOLD signal overlap with specific ROls. Indeed, with the lower spatial voxel resolution of functional scans, and as observed in our localizer validation experiment, significant BOLD signal can spread beyond intrinsic structural landmarks. Level 4 of the SARM hierarchy should be rather safe for most $\mathrm{fMRI}$ analyses, but the higher the level, the more careful one has to be to check the quality of $\mathrm{fMRI}$ registration.

The SARM is intended to support subcortical localization for a host of neuroimaging datasets (i.e., fMRI, PET, or diffusion imaging). While studies using a small number of macaque 1044 subjects can rely on directly comparing their signal location to print atlases, the SARM allows for 1045 morphing data from multiple subjects to an MRI-based atlas (and vice versa) to conduct group1046 level analyses using any neuroimaging modalities. The explosion of community data sharing 1047 (Milham et al., 2020) and multi-center NHP fMRI projects should, therefore, highly benefit from 1048 this resource. Furthermore, the SARM itself was conceived and developed within the context of 1049 the PRIMatE-Data Exchange (PRIME-DE) and will greatly benefit from usage-based feedback 1050 from the community. In addition to its utility for data analysis and identifying structures, the 1051 SARM in stereotaxic space has the potential to aid with surgical planning in studies involving 1052 tracer injections, drug injections, lesions, electrophysiology, optogenetics, and electrical 1053 stimulation, including deep brain stimulation (e.g., Ewerts et al., 2017). Beyond studies 
1054

1055

1056

1057

1058

1059

1060

1061

1062

1063

1064

1065

1066

1067

1068

1069

1070

1071

1072

1073

1074

1075

1076

1077

1078

1079

1080

1081

1082

1083

conducted solely in macaques, providing that homology and nomenclature equivalencies can be reliably established, future harmonized versions of the SARM, CHARM and human atlas counterparts could further help in comparing structural and functional organization between macaques and humans at a broad scale (Mantini et al., 2012). In this context, the detail of the SARM may be especially important, for example for DBS, where experimentally exploring spatially distinct neurostimulating sites could help explain variations in clinical results (Ewerts et al., 2017).

\section{Conclusion}

We have presented a new subcortical atlas for the rhesus macaque: the Subcortical Atlas of the Rhesus Macaque (SARM). Based primarily on the high-resolution MRI of a single subject and comparison with histological materials, this atlas provides the most detailed subcortical parcellation to date and is the first specifically applied to the subcortex available in a digital format for use by the MRI and general neuroscience community. We provide a specific use case and working examples of the use of this atlas within two popular fMRI analysis software packages. The SARM is part of a larger push in the NHP neuroimaging community to share data and resources. Information on the SARM and other macaque resources may be found at the PRIME-RE (Messinger et al., this issue).

\section{CRediT author statement}

Renée Hartig: conceptualization; resources; methodology; software; formal analysis; visualization; writing - original draft; writing - reviewing \& editing

Daniel Glen: resources; methodology; software; formal analysis; visualization; data curation; writing - original draft; writing - reviewing \& editing

Benjamin Jung: resources; methodology; software; formal analysis; visualization; writing original draft; writing - reviewing \& editing

Nikos K. Logothetis: resources; methodology; funding acquisition.

George Paxinos: resources; methodology; writing - reviewing \& editing

Eduardo Garza-Villarreal: resources; methodology; visualization; software; formal analysis; writing - original draft; writing - reviewing \& editing

Adam Messinger: conceptualization; resources; methodology; software; visualization; writing original draft; writing - reviewing \& editing 
Henry C. Evrard: conceptualization; resources; methodology; visualization; writing - original draft; writing - reviewing \& editing

\section{Acknowledgments}

EAGV would like to thank Gabriel A. Devenyi for his feedback and support, as well as the Laboratorio Nacional de Visualización Científica Avanzada (LAVIS) for the use of their computer cluster and the Laboratorio Nacional de Imagenología por Resonancia Magnética (LANIREM). $\mathrm{RH}$ and HCE would like to thank Michael Beyerlein and Thomas Steudel for their technical assistance with 7T imaging, and Yusuke Murayama for discussions on the visual flicker paradigm. This work was funded in part by the Max Planck Society and by the Intramural Research Program of the NIMH and NINDS (ZIA MH002918 and ZICMH002888).

\section{Declaration of competing interest}

The authors report no competing interest.

1100

1101

1102

1103

1104

1105

1106

1107

1108

1109

1110

1111

1112

\section{References-}

Accolla, E.A., Dukart, J., Helms, G., Weiskopf, N., Kherif, F., Lutti, A., ... Draganski, B., 2014. Brain tissue properties differentiate between motor and limbic basal ganglia circuits. Human Brain Mapping. 35, 5083-5092. doi:10.1002/hbm.22533

Amaral, D.G., Price, J.L., Pitkänen, A., Carmichael, S.T., 1992 Anatomical organization of the primate amygdaloid complex. In: The amygdala: Neurobiological aspects of emotion, memory, and mental dysfunction (Aggleton JP, ed.) pp. 1-66. New York, NY: Wiley-Liss.

Arsenault, J.T and Vanduffel, W., 2019. Ventral midbrain stimulation induces perceptual learning and cortical plasticity in primates. Nat Comm. 10, 3591. doi.org/10.1038/s41467-019-11527-9

Ashburner, J., 2007. A fast diffeomorphic image registration algorithm. Neurolmage. 38, 95-113.

Avants, B.B., Tustison, N.J., Stauffer, M., Song, G., Wu, B., Gee, JC., 2014. The Insight ToolKit Image Registration Framework. Frontiers in Neuroinformatics. 8: 44. doi.org/10.3389/fninf.2014.00044

Baker, J.T., Patel, G.H., Corbetta, M., Snyder, L.H., 2006. Distribution of Activity across the Monkey Cerebral Cortical Surface, Thalamus and Midbrain during Rapid, Visually Guided Saccades. Cerebral Cortex. 16(4), 447-459. doi: 10.1093/cercor/bhi124 
Bakker, R., Tiesinga, P., Kötter, R., 2015. The Scalable Brain Atlas: instant web-based access to public brain atlases and related content. Neuroinformatics. 13, 353-366. https://doi.org/10.1007/s12021-014-9258-x

Calabrese, E., Badea, A., Coe, CL., Lubach, GR., Shi, Y., Styner, MA., Johnson GA., 2015. A diffusion tensor MRI atlas of the postmortem rhesus macaque brain. Neurolmage. 117, 408-416.

Calzavara, R., Zappala, A., Rozzi, S., Matelli, M., Luppino G., 2005. Neurochemical Characterization of the Cerebellar-Recipient Motor Thalamic Territory in the Macaque Monkey. European Journal of Neuroscience 21(7), 1869-94. doi.wiley.com/10.1111/j.1460-9568.2005.04020.x

Carmichael, S.T. and Price J.L., 1994. Architectonic subdivision of the orbital and medial prefrontal cortex in the macaque monkey. Journal of Comparative Neurology 346(3), 366-402.

Chakraverty, M.M., Bertand, G., Hodge, C.P., Sadikot, A.F., Collins, D.L., 2006. The creation of a brain atlas for image guided neurosurgery using serial histological data. Neurolmage. 30(2), 359-376.

Cox, R.W., 1996. AFNI: Software for Analysis and Visualization of Functional Magnetic Resonance Neuroimages. Computers and Biomedical Research, 29, 162-173.

Evrard, H.C. Logothetis, N.K., Craig, A.D., 2014. Modular Architectonic Organization of the Insula in the Macaque Monkey. Journal of Comparative Neurology 522(1), 64-97.

Evrard, H.C., Forro, T., Logothetis, N.K., 2012. Von Economo Neurons in the Anterior Insula of the Macaque Monkey. Neuron. 74(3), 482-489. doi.org/10.1016/j.neuron.2012.03.003

Evrard, H.C, and Craig A.D., 2008. Retrograde Analysis of the Cerebellar Projections to the Posteroventral Part of the Ventral Lateral Thalamic Nucleus in the Macaque Monkey. Journal of Comparative Neurology 508(2), 286-314.

Ewert, S., Plettig, P., Li, N., Chakravarty, M.M., Collins, D.L., Herrington, T.M., Kuehn, A.A., Horn, A., 2017. Toward defining deep brain stimulation targets in MNI space: A subcortical atlas based on multimodal MRI, histology and structural connectivity. Neurolmage. 170, 271-282. doi: 10.1016/j.neuroimage.2017.05.015

Fox, A., Oler, J.A., Shackman, A.J., Shelton, S.E., Raveendran, M., McKay, D.R., Converse, A.K., Alexander, A., Davidson, R.J., Blangero, J., Rogers, J., Kalin, N.H., 2015. Intergenerational neural mediators of early-life anxious temperament. PNAS. 112(29), 9118-9122. https://doi.org/10.1073/pnas.1508593112

Feng, L., Jeon, T., Yu, Q., Ouyang, M., Peng, Q., Mishra, V., Pletikos, M., Sestan, N., Miller, MI., Mori, S., Hsiao, S., Liu, S., Huang, H., 2017. Population-averaged macaque brain atlas with high-resolution ex vivo DTI integrated into in vivo space. Brain Struct Funct. 222(9), 4131-4147. doi: 10.1007/s00429-017-1463-6

Haber, S.N., Adler, A., Bergman, H., 2012. The Basal Ganglia. In Mai J.K. and Paxinos G. (Eds), The Human Nervous System, 3rd Ed. Academic Press. 
Horsley, V. and Clarke, R.H., 1908. THE STRUCTURE AND FUNCTIONS OF THE CEREBELLUM EXAMINED BY A NEW METHOD. Brain. 31(1), 45-124. https://doi.org/10.1093/brain/31.1.45

Jung, B., Taylor, P.A., Seidlitz, J., Sponheim, C., Perkins, P., Ungerleider, L.G., Glen, D., Messinger, A., This issue. A comprehensive macaque fMRI pipeline and hierarchical atlas. Neurolmage.

Klink, P.C., Aubry, J.-F., Ferrera, V., Fox, A., Froudist-Walsh, S., Jarraya, B., Konofagou, E., Krauzlis, R., Messinger, A., Mitchell, A.S., Ortiz-Rios, M., Oya, H., Premereur, E., Roberts, A., Roe, A., Rushworth, M.F.S., , Sallet, J., Schmid, M.C., Schroeder, C.E., Tasserie, J., Tsao, D., Uhrig, L., Vanduffel, W., Wilke, M., Kagan, I., Petkov, C.I. Combined Brain Perturbation and Neuroimaging in Non-human Primates. Neurolmage. This issue.

Kochunov, P., Lancaster, J.L., Thompson, P., Woods, R., Mazziotta, J., Hardies, J., Fox, P., 2001. Regional Spatial Normalization: Toward an Optimal Target. Journal of Computer Assisted Tomography. 25(5), 805-816.

Kriegeskorte, N., Simmons, W.K., Bellgowan, P.S., Baker, C.I., 2009. Circular analysis in systems neuroscience: the dangers of double dipping. Nat Neurosci. 12(5), 535-540. doi:10.1038/nn.2303

Logothetis, N.K., Eschenko, O., Murayama, Y., Augath, M., Steudel, T., Evrard, H.C., ... Oeltermann, A., 2012. Hippocampal-cortical interaction during periods of subcortical silence. Nature, 491(7425), 547-553. https://doi.org/10.1038/nature11618

Logothetis, N.K., Augath, M., Murayama, Y., Rauch, A., Sultan, F., Goense, J., ... Merkle, H., 2010. The effects of electrical microstimulation on cortical signal propagation. Nature Neuroscience, 13(10), 1283-1291. https://doi.org/10.1038/nn.2631

Logothetis, N.K., Guggenberger, H., Peled, S., Pauls, J., 1999. Functional imaging of the monkey brain. Nature Neurosci. 2, 555-562.

Mai, J.K. and Forutan, F., 2012. Thalamus. In Mai, J.K. and Paxinos, G. (Eds), The Human Nervous System, 3rd Ed. Academic Press.

Mai, J.K. and Paxinos, G., 2012. The Human Nervous System, 3rd Ed. Academic Press.

Mantini, D., Hasson, U., Bett, V., Perrucci, M.G., Romani, G.L., Corbetta, M., Orban G.A., Vanduffel, W., 2012. Interspecies activity correlations reveal functional correspondence between monkey and human brain areas. Nature Methods. 9(3), 277-282. https://doi.org/10.1038/nmeth.1868

Martin, R.F. and Bowden, D.M., 2000. Primate Brain Maps: Structure of the Macaque Brain. Elsevier Science. University of Washington, U.S.A.

Martin, R.F, Bowden, D.M, Wu, J, Dubach, M.F, Robertson, J.E., 2000. Primate Brain Maps: Structure of the Macaque Brain. Elsevier, Amsterdam, Netherlands. 
Messinger, A., et al. submitted. A collaborative resource platform for non-human primate neuroimaging. Neurolmage. This issue.

Milham, M., Petkov, C.I., Margulies, D.S., Schroeder, C.E., Basso, M.A., Belin, P., Fair, D.A., Fox, A., Kastner, S., Mars, R.B., Messinger, A., Poirier, C., Vanduffel, W., Van Essen, D.C., Alvand, A., Becker, Y., Ben Hamed, S., Benn, A., Bodin, C., Boretius, S., Cagna, B., Coulon, O., El-Gohary, S.H., Evrard, H., Forkel, S.J., Friedrich, P., FroudistWalsh, S., Garza-Villarreal, E.A., Gao, Y., Gozzi, A., Grigis, A., Hartig, R., Hayashi, T., Heuer, K., Howells, H., Ardesch, D.J., Jarraya, B., Jarrett, W., Jedema, H.P., Kagan, I., Kelly, C., Kennedy, H., Klink, P.C., Kwok, S.C., Leech, R., Liu, X., Madan, C., Madushanka, W., Majka, P., Mallon, A.-M., Marche, K., Meguerditchian, A., Menon, R.S., Merchant, H., Mitchell, A., Nenning, K.-H., Nikolaidis, A., Ortiz-Rios, M., Pagani, M., Pareek, V., Prescott, M., Procyk, E., Rajimehr, R., Rautu, I.-S., Raz, A., Roe, A.W., Rossi-Pool, R., Roumazeilles, L., Sakai, T., Sallet, J., García-Saldivar, P., Sato, C., Sawiak, S., Schiffer, M., Schwiedrzik, C.M., Seidlitz, J., Sein, J., Shen, Z., Shmuel, A., Silva, A.C., Simone, L., Sirmpilatze, N., Sliwa, J., Smallwood, J., Tasserie, J., Thiebaut de Schotten, M., Toro, R., Trapeau, R., Uhrig, L., Vezoli, J., Wang, Z., Wells, S., Williams, B., Xu, T., Xu, A.G., Yacoub, E., Zhan, M., Ai, L., Amiez, C., Balezeau, F., Baxter, M.G., Blezer, E.L.A., Brochier, T., Chen, A., Croxson, P.L., Damatac, C.G., Dehaene, S., Everling, S., Fleysher, L., Freiwald, W., Griffiths, T.D., Guedj, C., HadjBouziane, F., Harel, N., Hiba, B., Jung, B., Koo, B., Laland, K.N., Leopold, D.A., Lindenfors, P., Meunier, M., Mok, K., Morrison, J.H., Nacef, J., Nagy, J., Pinsk, M., Reader, S.M., Roelfsema, P.R., Rudko, D.A., Rushworth, M.F.S., Russ, B.E., Schmid, M.C., Sullivan, E.L., Thiele, A., Todorov, O.S., Tsao, D., Ungerleider, L., Wilson, C.R.E., Ye, F.Q., Zarco, W., Zhou, Y., 2020. Accelerating the Evolution of Nonhuman Primate Neuroimaging. Neuron 105, 600-603. doi.org/10/ggvm7d

1218

1219

1220

1221

1222

1223

1224

1225

1226

1227

1228

1229

1230

1231

1232

Milham, M.P., Ai, L., Koo, B., Xu, T., Amiez, C., Balezeau, F., Baxter, M.G., Blezer, E.L.A., Brochier, T., Chen, A., Croxson, P.L., Damatac, C.G., Dehaene, S., Everling, S., Fair, D.A., Fleysher, L., Freiwald, W., Froudist-Walsh, S., Griffiths, T.D., Guedj, C., HadjBouziane, F., Ben Hamed, S., Harel, N., Hiba, B., Jarraya, B., Jung, B., Kastner, S., Klink, P.C., Kwok, S.C., Laland, K.N., Leopold, D.A., Lindenfors, P., Mars, R.B., Menon, R.S., Messinger, A., Meunier, M., Mok, K., Morrison, J.H., Nacef, J., Nagy, J., Rios, M.O., Petkov, C.I., Pinsk, M., Poirier, C., Procyk, E., Rajimehr, R., Reader, S.M., Roelfsema, P.R., Rudko, D.A., Rushworth, M.F.S., Russ, B.E., Sallet, J., Schmid, M.C., Schwiedrzik, C.M., Seidlitz, J., Sein, J., Shmuel, A., Sullivan, E.L., Ungerleider, L., Thiele, A., Todorov, O.S., Tsao, D., Wang, Z., Wilson, C.R.E., Yacoub, E., Ye, F.Q., Zarco, W., Zhou, Y., Margulies, D.S., Schroeder, C.E., 2018. An Open Resource for Non-human Primate Imaging. Neuron 100, 61-74.e2. doi.org/10/gffxtn

Molfese, P.J., Glen, D., Mesite, L., Cox, R.W., Hoeft, F., Frost, S.J., Mencl, W.E., Pugh, K., \& Bandettini, P.A. (Accepted). The Haskins pediatric atlas: An MRI-based pediatric template and atlas. Pediatric Radiology.

Morel, A, Magnin, M, Jeanmonod, D., 1997. Multiarchitectonic and stereotactic atlas of the human thalamus. J Comp Neurol. 387, 588-630.

Murris, S.R., Arsenault, J.T., Vanduffel, W., 2020. Frequency- and State-Dependent Network Effects of Electrical Stimulation Targeting the Ventral Tegmental Area in Macaques. Cerebral Cortex. 00, 1-16. doi.org/10.1093/cercor/bhaa007 
1238

1239

1240

1241

1242

1243

1244

1245

1246

1247

1248

1249

1250

1251

1252

1253

1254

1255

1256

1257

1258

1259

1260

1261

1262

1263

1264

1265

1266

1267

1268

1269

1270

1271

1272

1273

1274

1275

1276

Nassi, J.J., Avery M.C., Cetin, A.H., Roe, A.W., Reynolds, J.H., 2015. Optogenetic Activation of Normalization in Alert Macaque Visual Cortex. Neuron 86(6): 1504-1517. doi.org/10.1016/j.neuron.2015.05.040

Noonan, M.P., Sallet, J., Mars, R.B., Neubert, F.X., O'Reilly, J.X., Andersson, J.L., Mitchell, A.S., Bell, A.H., Miller, K.L., Rushworth, M.F.S., 2014. A Neural Circuit Covarying with Social Hierarchy in Macaques. PLoS Biol 12(9), e1001940. doi.org/10.1371/journal.pbio.1001940

Oler, J.A., Birn, R.M., Patriat, R., Fox, A.S., Shelton, S.E., Burghy, C.A., Stodola, D.E., Essex, M.J., Davidson, R.J., Kalin, N.H., 2012. Evidence for coordinated functional activity within the extended amygdala of non-human and human primates. Neuroimage 61 , 1059-1066. doi:10.1016/j.neuroimage.2012.03.045 pmid:22465841

Olszewki, J., 1952. The Thalamus of the Macaca Mulatta: An Atlas for Use with the Stereotaxic Instrument. Karger, New York.

Ortiz-Rios, M., Kusmierek, P., DeWitt, I., Archakov, D., Azevedo F.A.C., Sams, M., Jaaskelainen I.P., Keliris, G.A., Rauschecker, J.P., 2015. Functional MRI of the Vocalization-Processing Network in the Macaque Brain. Frontiers in Neuroscience 9, 113. doi.org/10.3389/fnins.2015.00113

Pauli, W.M., Nili, A.N., Tyszka, J.M., 2018. A high-resolution probabilistic in vivo atlas of human subcortical brain nuclei. Scientific Data. 5, 180063.

Paxinos, G., Petrides, M., Evrard, H.C., in preparation. The Rhesus Monkey Brain in Stereotaxic Coordinates $4^{\text {th }}$ Ed.. Elsevier.

Paxinos, G., Huang, X.-F., Petrides, M., Toga, A.W., 2009. The Rhesus Monkey Brain in Stereotaxic Coordinates, 2nd Edn. Elsevier Academic Press, San Diego, U.S.A.

Pipitone, J., Park, M.T.M., Winterburn, J., Lett, T.A., Lerch, J.P., Pruessner, J.C., Lepage, M., Voineskos, A.N., Chakravarty, M.M., the Alzheimer's Disease Neuroimaging Initiative., 2014. Multi-atlas segmentation of the whole hippocampus and subfields using multiple automatically generated templates. Neurolmage. 101, 494-512.

Puelles, L., Harrison, M., Paxinos, G., Watson, C., 2013. A developmental ontology for the mammalian brain based on the prosomeric model. Trends Neurosci 36, 570-8.

Quan, Z., Gao, Y., Qu, S., Wang, X., Friedman, R.M., Chernov, M.M., Kroenke, C.D., Roe, A.W., Zhang, X., 2020. A 16-Channel Loop Array for in Vivo Macaque Whole-Brain Imaging at $3 \mathrm{~T}$. Magnetic Resonance Imaging. 68, 167-72.

Reveley, C., Gruslys, A., Ye, F.Q., Glen, D., Samaha, J., Russ, B.E., ... Saleem, K.S., 2017. Three-Dimensional Digital Template Atlas of the Macaque Brain. Cerebral Cortex, 1-15. doi.org/10.1093/cercor/bhw248

Rohlfing, T., Kroenke, C.D., Sullivan, E.V., Dubach, M.F., Bowden, D.M., Grant, K.A., Pfefferbaum A., 2012. The INIA19 Template and NeuroMaps Atlas for Primate Brain Image Parcellation and Spatial Normalization. Frontiers in Neuroinformatics. 6:27. doi.org/10.3389/fninf.2012.00027 
Saad, Z.S., Reynolds, R.C., Argall, B., Japee, S., Cox, R.W., 2004. SUMA: an interface for surface-based intra- and inter-subject analysis with AFNI. 2nd IEEE International Symposium on Biomedical Imaging: Nano to Macro (IEEE Cat No. 04EX821). 2, 15101513. https://doi.org/10.1109/ISBI.2004.1398837

Saad, Z.S., Glen, D.R., Chen, G., Beauchamp, M.S., Desai, R., Cox, R.W., 2009. A new method for improving functional-to-structural MRI alignment using local Pearson correlation. Neurolmage. 44, 839-848. 10.1016/j.neuroimage.2008.09.037

Saleem, K.S. and Logothetis, N.K. 2012., A Combined MRI and Histology Atlas of the Rhesus Monkey Brain in Stereotaxic Coordinates, 2nd Edition. Academic Press.

Saper, C.J., 2012. Hypothalamus. In Mai, J.K. and Paxinos, G. (Eds), The Human Nervous System, 3rd Ed. Academic Press.

Seidlitz, J., Sponheim, C., Glen, D., Ye, F.Q., Saleem, K.S., Leopold, D.A., Ungerleider, L., Messinger, A., 2018. A population MRI brain template and analysis tools for the macaque. Neurolmage. 170, 121-131. doi.org/10.1016/j.neuroimage.2017.04.063

Stauffer, W.R., Lak, A., Yang, A., Borel, M., Paulsen, O., Boyden, E.S., Schultz, W., 2016. Dopamine Neuron-Specific Optogenetic Stimulation in Rhesus Macaques. Cell 166(6), 1564-1571.e6.

Stefanacci, L. and Amaral, D.G., 2000. Topographic Organization of Cortical Inputs to the Lateral Nucleus of the Macaque Monkey Amygdala: A Retrograde Tracing Study. Journal of Comparative Neurology. 421(1), 52-79.

Tustison, N.J., Avants, B.B., Cook, P.A., Zheng, Y., Egan, A., Yushkevich, P.A., Gee, J.C., 2010. N4ITK: Improved N3 Bias Correction. IEEE Transactions on Medical Imaging. 29(6), 1310-20. 


\section{Supplementary Material}

\section{Supplementary Figures:}
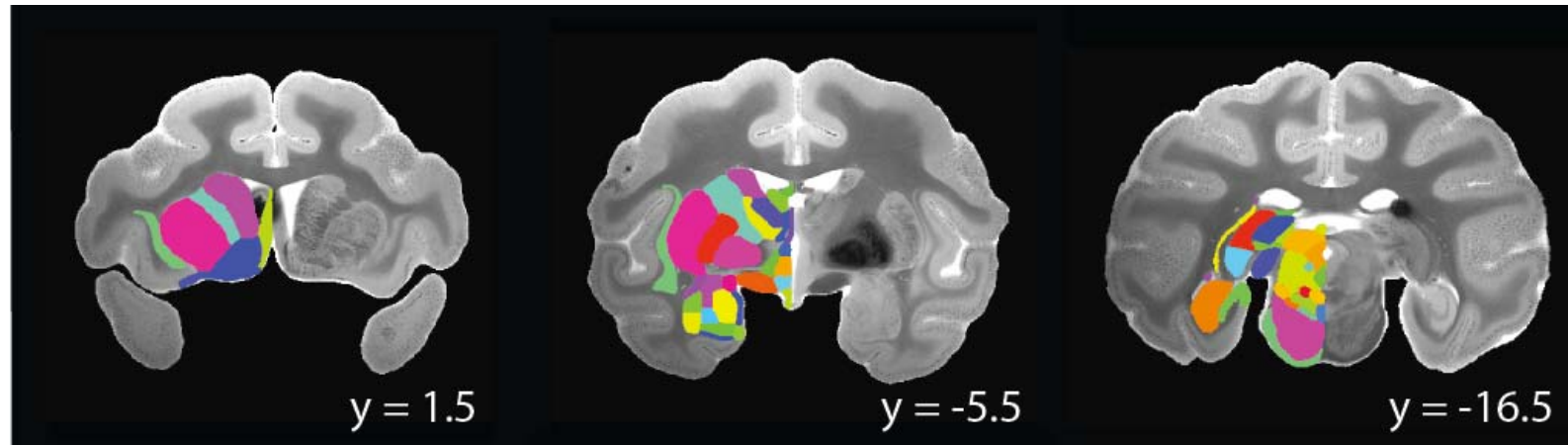

$$
y=1.5
$$

$$
y=-16.5
$$
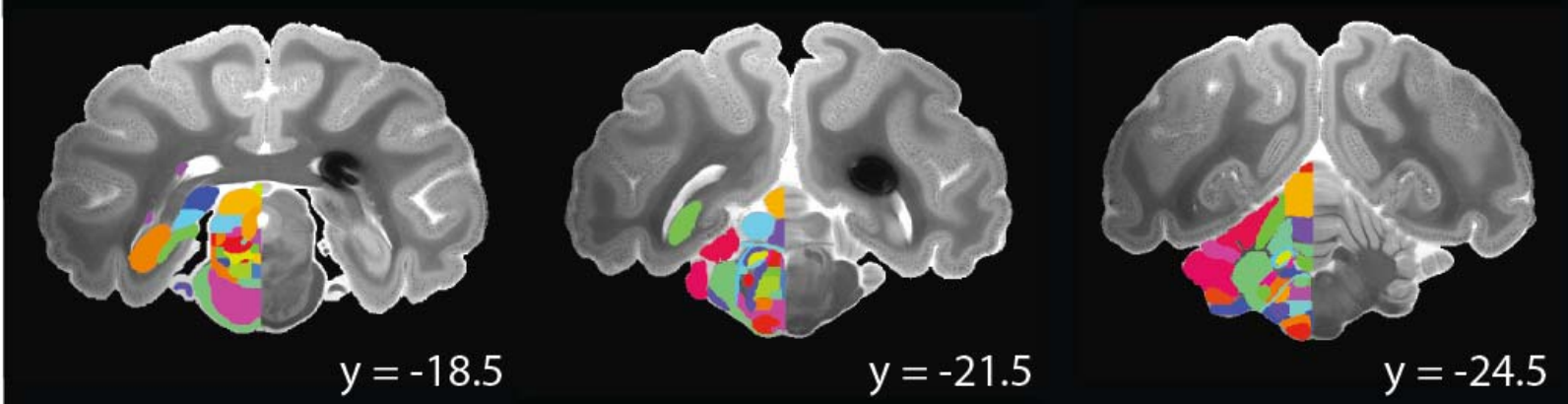

Figure S1. The subcortical atlas in G12 space. Coronal sections from subject G12 are shown with the subcortical parcellation overlaid for the left side regions. The high-resolution ex vivo scan was reoriented from its original orientation to a standard orientation. Coordinates are listed in $\mathrm{mm}$ in the individual subject's native space, left hemisphere shown left. 

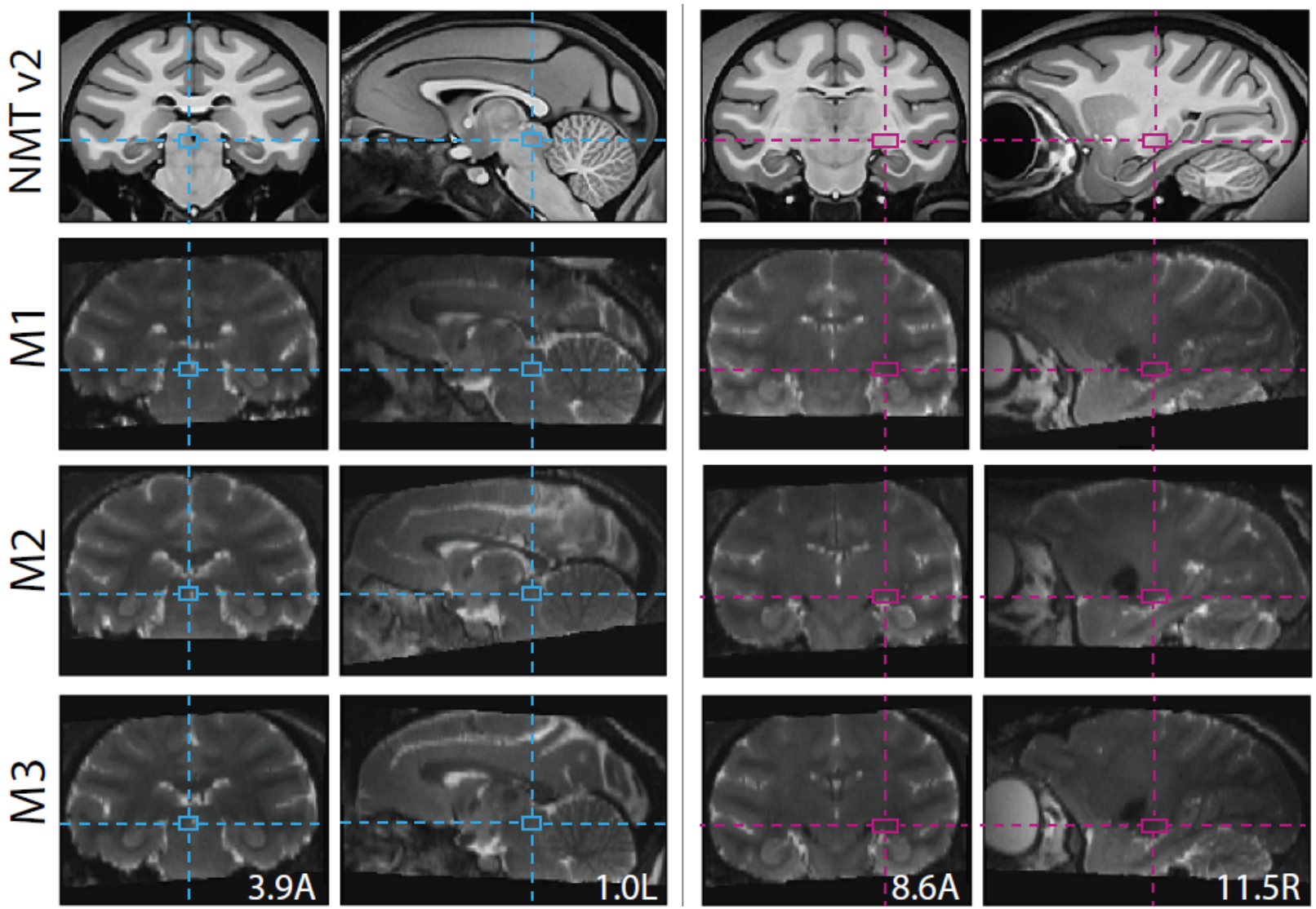

Figure S2. Nonlinear registration of three rhesus macaques to the NMT v2 template. Crosshairs intersecting at two subcortical regions: the left periaqueductal gray nucleus (PAG; left panels) in the mesencephalon and the right dorsal lateral geniculate nucleus (DLG, right panels) of the thalamus. The depth of all alignment boxes is $11.6 \mathrm{~S}$ (stereotaxic coordinates reported in $\mathrm{mm}$ from the ear bar zero; EBZ). The coronal and sagittal sections show the correspondence between the T1-weighted NMT v2 and the T2-weighted single-subject anatomical scans from macaque monkeys M1-M3 after Dartels-based 